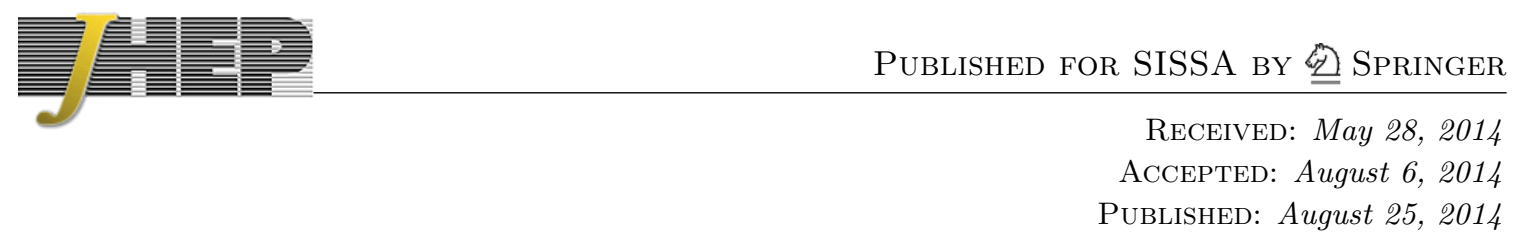

\title{
F-theory on genus-one fibrations
}

\section{Volker Braun and David R. Morrison}

University of Oxford, Andrew Wiles Building, Radcliffe Observatory Quarter,

Woodstock Road, Oxford, OX2 6GG, U.K.

Departments of Mathematics and Physics, University of California Santa Barbara,

Santa Barbara, CA 93106, U.S.A.

E-mail: volker.braun@maths.ox.ac.uk, drm@math.ucsb.edu

ABSTRACT: We argue that M-theory compactified on an arbitrary genus-one fibration, that is, an elliptic fibration which need not have a section, always has an F-theory limit when the area of the genus-one fiber approaches zero. Such genus-one fibrations can be easily constructed as toric hypersurfaces, and various $\mathrm{SU}(5) \times \mathrm{U}(1)^{n}$ and $E_{6}$ models are presented as examples. To each genus-one fibration one can associate a $\tau$-function on the base as well as an $\mathrm{SL}(2, \mathbb{Z})$ representation which together define the IIB axio-dilaton and 7-brane content of the theory. The set of genus-one fibrations with the same $\tau$-function and $\operatorname{SL}(2, \mathbb{Z})$ representation, known as the Tate-Shafarevich group, supplies an important degree of freedom in the corresponding F-theory model which has not been studied carefully until now.

Six-dimensional anomaly cancellation as well as Witten's zero-mode count on wrapped branes both imply corrections to the usual F-theory dictionary for some of these models. In particular, neutral hypermultiplets which are localized at codimension-two fibers can arise. (All previous known examples of localized hypermultiplets were charged under the gauge group of the theory.) Finally, in the absence of a section some novel monodromies of Kodaira fibers are allowed which lead to new breaking patterns of non-Abelian gauge groups.

Keywords: F-Theory, Differential and Algebraic Geometry

ARXIV EPRINT: 1401.7844 


\section{Contents}

1 Introduction 1

2 From genus-one fibrations to elliptic fibrations 4

3 Singularities of Jacobian fibrations $\quad 8$

4 Fiberwise duality with M-theory $\quad 10$

$\begin{array}{lll}4.1 & \text { Review of the F/M duality } & 10\end{array}$

4.2 Fiberwise duality 11

$\begin{array}{lll}4.3 & \text { Tate-Shafarevich group } & 12\end{array}$

4.4 Relation with discrete torsion 12

5 Moduli of genus-one fibrations 13

$\begin{array}{lll}5.1 & \text { Weierstrass hypersurface } & 13\end{array}$

$\begin{array}{ll}5.2 \text { Elliptic fibration with a toric SU(5) } & 15\end{array}$

$\begin{array}{ll}5.3 \text { Adding a single U(1) } & 16\end{array}$

$\begin{array}{lll}5.4 \mathrm{U}(1) \text { charge assignments } & 18\end{array}$

5.5 Three U(1)'s 20

5.6 No section 20

6 Gravitational anomaly cancellation $\quad 22$

7 Triality of $\boldsymbol{E}_{6}$ and monodromies $\quad 24$

$\begin{array}{ll}7.1 & \text { Unbroken gauge group }\end{array}$

$\begin{array}{lll}7.2 & \text { Alternative gauge groups } & 25\end{array}$

$\begin{array}{ll}7.3 & \text { The non-split case }\end{array}$

$\begin{array}{ll}7.4 & \text { Starting at the other end } \\ 7.5 & 28\end{array}$

7.5 A novel monodromy effect 31

7.6 Alternative limits 34

8 The Shioda-Tate(-Wazir) formula 36

$\begin{array}{ll}\text { A Representation theory in sage } & 38\end{array}$

\section{Introduction}

The original F-theory construction [1-3] produces a non-perturbative type IIB string vacuum by using a multi-valued function " $\tau$ " with an $\mathrm{SL}(2, \mathbb{Z})$ symmetry to specify the behavior of the two scalar fields in type IIB string theory. This function $\tau$ is defined on the 
complement of a subvariety $\Delta$ in a compact complex manifold $B$, the base of the F-theory fibration. Moreover, the $\tau$-function has a specific asymptotic behavior near the components of the discriminant $\Delta$, measured by an $\operatorname{SL}(2, \mathbb{Z})$-valued representation of the fundamental group of $B-\Delta$. The components of $\Delta$ serve as sources for the Ramond-Ramond scalar field, i.e., as 7-branes in the theory.

The basic F-theory construction comes along with F-theory/M-theory duality: if Ftheory is further compactified on a circle, the resulting theory should be dual to M-theory compactified on a Calabi-Yau variety $Y$ which is fibered over $B$ by curves of genus one, with the curves becoming singular over $\Delta$, and with the function $b \mapsto \tau(b)$ corresponding to the ratio of periods of the holomorphic 1-form on the fiber over $b$. As the area of the genus-one curves shrinks, the dual circle expands; the zero-area limit corresponds to decompactification to the original F-theory model. This duality arises by a fiberwise application of an M-theory/Type IIB duality $[4,5]$.

The Calabi-Yau varieties $Y$ whose M-theory compactifications have F-theory limits should therefore admit genus-one fibrations $Y \rightarrow B$, i.e., maps $\pi: Y \rightarrow B$ whose general fiber is a curve of genus one. Traditionally in the F-theory literature one has demanded that $\pi: Y \rightarrow B$ have a section but we relax that requirement in this paper. We do, however, require that the fibration $\pi$ is flat, i.e., that all fibers of $\pi$ are one-dimensional. (Otherwise, tensionless strings will give additional massless degrees of freedom in addition to the desired low-energy effective Yang-Mills theory.)

Since $Y$ is an algebraic variety whenever $\operatorname{dim} Y \geq 3$, it must have an embedding into projective space with some equations defining the image; the existence of the genus-one fibration means that those equations define a family of curves of genus one parametrized by $B$. In particular, these equations can be regarded as defining a curve of genus one whose defining equations have coefficients which are locally defined functions on $B$. By allowing denominators in the coefficients, we can instead think of this as a curve of genus one whose defining equations have coefficients chosen from the field $K$ of rational functions on $B$. The equations for various open subsets of $B$ will all induce the same equation with coefficients in $K$.

Algebraic geometers and number theorists have long studied algebraic curves whose defining equations have coefficients in a field $K$ that may not be algebraically closed. If a curve of genus one over $K$ has a point on it with coefficients in $K$, then the curve is called an elliptic curve over $K$ and its points form an Abelian group. When $K$ is the function field of a base manifold $B$, the points with coefficients in $K$ correspond to rational sections of the genus-one fibration; thus, a genus-one fibration is an elliptic fibration exactly when there is a rational section.

Every algebraic curve $C$ of genus one over $K$ has an associated elliptic curve $J(C)$ over $K$ known as the Jacobian of $C$ as we will review in section 2. Geometrically, the points on the Jacobian represent line bundles of degree zero on $C$, and since there is a distinguished line bundle (the trivial line bundle) the Jacobian has a distinguished point. Moreover, since the set of line bundles of degree zero forms a group (under tensor product), the group structure on $J(C)$ is evident. 
If $Y$ is a Calabi-Yau surface or threefold with a genus-one fibration, then the (compactified) Jacobian of the fibration determines an associated Calabi-Yau variety ${ }^{1} J(Y)$ with an elliptic fibration [6-8]. Moreover, the multi-valued $\tau$ functions and $\mathrm{SL}(2, \mathbb{Z})$ representations for $Y \rightarrow B$ and $J(Y) \rightarrow B$ are identical. (Even the discriminant loci for the fibrations are identical as subvarieties of $B$ [9].) For this reason, one should expect that the F-theory limits of M-theory compactified on $Y$ and on $J(Y)$ are identical. It has often been asserted - particularly by the second author of this paper - that this fact makes the study of non-elliptic genus-one fibrations superfluous for F-theory. However, as we will show in this paper, the compactified Jacobian $J(Y)$ typically has singularities for a nonsingular genusone fibered Calabi-Yau threefold $Y$ without a section. The singularities in question are "Q্-factorial terminal" singularities (the simplest example being a conifold with no small resolution) which have the property that any blowup of them introduces zeros into the holomorphic 3-form.

The presence of such singularities on $J(Y)$ at first leads one to expect that M-theory cannot be compactified on this space. However, since the nonsingular space $Y$ has a good F-theory limit and shares the same $\tau$-function and monodromy representation with $J(Y)$, such an interpretation would be rather puzzling. To resolve this puzzle, one should remember that when the M-theory 3-form has torsion flux, there can be so-called "frozen" singularities linked to that flux which are not resolved in the M-theory compactification, and which do not contribute to the M-theory gauge group in the standard way $[10,11]$. Although a complete understanding of such torsion fluxes awaits a better understanding of the M-theory 3-form, we shall argue in this paper that this is indeed the correct explanation.

In practice, it is often easier to study $Y$ than $J(Y)$, so these genus-one fibrations are not superfluous. In fact, as we will see in detail in section 5, and as had been earlier observed in [12], this phenomenon already happens for Calabi-Yau threefold hypersurfaces in rather simple toric varieties. Still, one might ask how F-theory on genus-one fibrations is really different from the familiar case of elliptic fibrations. In this paper, we will point out the following two:

- There can be massless fields localized on codimension-two fibers. In fact, subtleties in the interpretation of the F-theory limit for a genus-one fibration without a section were observed already in [13] which considered a "degree two" case in the language of this paper. In section 6 we will explain the origin of these massless fields. In particular, the existence of such fields corrects the hypermultiplet moduli count of 6-d F-theory compactifications. After taking this correction into account, the gravitational anomaly is again canceled.

- There are more possibilities for the monodromies of the Kodaira fiber over a discriminant component. In fact, if one requires a section then one of the $\mathbb{P}^{1}$-components with multiplicity 1 (corresponding to a node of the Dynkin diagram with Dynkin label 1) must be fixed by the monodromy. This need not be the case if there is no

\footnotetext{
${ }^{1}$ This statement is presumably also true for Calabi-Yau fourfolds, but a mathematical proof has not yet been given.
} 
section, yielding new ways to break non-Abelian gauge symmetry by monodromies. For example, in section 7 we will discuss an example of a toric hypersurface where monodromy breaks $E_{6} \rightarrow G_{2}$. The Shioda-Tate(-Wazir) formula for the cohomology groups of elliptic fibrations naturally generalizes to genus-one fibrations with more general monodromies, see section 8 .

On the base $B$ of the elliptic fibration, the data defining the genus-one fibration $Y \rightarrow B$ is the $\tau$-function and $\mathrm{SL}(2, \mathbb{Z})$ representation (or equivalently, the compactified Jacobian $J(Y)$ ), together with a class in the Tate-Shafarevich group [14, 15] of isomorphism classes of elliptic fibrations with the same Jacobian. The Tate-Shafarevich group $\amalg_{B}(J(Y))$ can be described in in terms of certain sheaf cohomology groups on the base $B$ (which make it obvious that it is indeed a group). The simplest description $[9,16,17]$, which holds when $J(Y)$ and $B$ are both smooth, all fibers of the fibration are irreducible, ${ }^{2}$ and the section of $J(Y) \rightarrow B$ is regular, is

$$
\amalg_{B}(J(Y))=H^{1}(B, \mathcal{A}),
$$

where $\mathcal{A}$ is the sheaf of rational sections of $J(Y) \rightarrow B$. The interpretation in F-theory is of a degree of freedom which can be given an expectation value on a loop when compactifying F-theory to M-theory, resulting in a collection of M-theory vacua with the same F-theory limit $[11,18]$.

\section{From genus-one fibrations to elliptic fibrations}

Let us start by reviewing how to assign the multi-valued $\tau$ function to a genus-one fibration, that is, how the defining equations of the given fibration determine defining equations of the Jacobian fibration. (The latter can always be brought into Weierstrass form.) A CalabiYau variety $Y$ of dimension $k$ with a genus-one fibration always has an embedding into projective space when $k \geq 3 .^{3}$ Let us choose a line bundle $\mathcal{L}$ on $Y$ which is ample on the general fiber of the fibration, ${ }^{4}$ and let $d$ be the degree of $\mathcal{L}$ when restricted to the curves of genus one. That is, the associated curve $C$ of genus one over the function field $K$ has a line bundle $\mathcal{L}_{\mathcal{C}}$ of degree $d$. For small values of $d$, the properties of $\mathcal{L}_{\mathcal{C}}$ determine the structure of a birational model $\bar{Y}$ of $Y$. This determination is based on the Riemann-Roch theorem for $C$, which continues to hold even over a non-algebraically closed field. For a line bundle $\mathcal{M}$ of positive degree on a curve of genus one, $H^{1}(C, \mathcal{M}) \cong H^{0}\left(C, K_{C} \otimes \mathcal{M}^{-1}\right)^{*}$ which vanishes since $\operatorname{deg}\left(K_{C} \otimes \mathcal{M}^{-1}\right)=0-\operatorname{deg}(\mathcal{M})<0$. Thus,

$$
\operatorname{dim} H^{0}(C, \mathcal{M})=\chi(C, \mathcal{M})=\operatorname{deg}(\mathcal{M})-g+1=\operatorname{deg}(\mathcal{M})
$$

since $g=1$.

If $C$ has a line bundle $\mathcal{L}$ of degree 1 , this leads to "Weierstrass form" in the following way, as explained by Deligne [19] (see also $[8,20]) . H^{0}(C, \mathcal{L})$ has dimension one, so choose

\footnotetext{
${ }^{2}$ Note that this condition implies no non-Abelian gauge symmetry in the F-theory model; the description must be modified in more general cases.

${ }^{3}$ The case $k=2$ is special because a K3 surface may fail to be a projective variety.

${ }^{4}$ That is, the sections of some power of $\mathcal{L}$ give a map to projective space which is an embedding of the general fiber.
} 
a basis element $z$ for this space. $H^{0}\left(C, \mathcal{L}^{\otimes 2}\right)$ has dimension two and contains $z^{2}$; choose another element $x$ so that $z^{2}, x$ is a basis of this space. $H^{0}\left(C, \mathcal{L}^{\otimes 3}\right)$ has dimension three and contains $z^{3}$ and $x z$; choose another element $y$ so that $z^{3}, x z, y$ is a basis of this space. We can now enumerate the sections we know of various powers of $\mathcal{L}$ :

$$
\begin{aligned}
& H^{0}(C, \mathcal{L}) \quad \ni z \\
& H^{0}\left(C, \mathcal{L}^{\otimes 2}\right) \ni z^{2}, x \\
& H^{0}\left(C, \mathcal{L}^{\otimes 3}\right) \ni z^{3}, x z, y \\
& H^{0}\left(C, \mathcal{L}^{\otimes 4}\right) \ni z^{4}, x z^{2}, y z, x^{2} \\
& H^{0}\left(C, \mathcal{L}^{\otimes 5}\right) \ni z^{5}, x z^{3}, y z^{2}, x^{2} z, x y \\
& H^{0}\left(C, \mathcal{L}^{\otimes 6}\right) \ni z^{6}, x z^{4}, y z^{3}, x^{2} z^{2}, x y z, x^{3}, y^{2} .
\end{aligned}
$$

Since there are seven sections of $H^{0}\left(C, \mathcal{L}^{\otimes 6}\right)$, there must be a relation among them. As Deligne argues, since there is no relation among sections of lower powers of $\mathcal{L}$, the coefficients of $x^{3}$ and $y^{2}$ in the relation must be nonvanishing, and after rescaling we can assume those coefficients are 1 . Thus, we get an equation of the form

$$
y^{2}+a_{1} x y z+a_{3} y z^{3}=x^{3}+a_{2} x^{2} z^{2}+a_{4} x z^{4}+a_{6} z^{6}
$$

the long Weierstrass from. If the characteristic of $K$ is not 2 or 3 , as is true in our case, we arrive at an equation of the form

$$
y^{2}=x^{3}+f x^{2} z^{4}+g z^{6}
$$

by completing the square on $y$ and completing the cube on $x$. This is the Weierstrass form.

We can "clear denominators" in the coefficients $f, g \in K$ in the Weierstrass equation and take them to lie in the ring of functions on any subset of the base $B$. More generally, $f$ and $g$ can be taken as sections of appropriate line bundles over $B$, and this gives the most general Weierstrass forms of elliptic fibrations. Note that any genus-one fibration of degree 1 has a section (given by $z=0$ in the coordinates above); that is, it is an elliptic fibration.

There are similar stories for other low values of the degree $d$. First, one can analyze sections of the line bundle to determine the form of the equation of the curve $C$ of genus one. Then, the geometric construction of the Jacobian fibration can be studied algebraically, resulting in a formula for the equation of the Jacobian $J(C)$, given the equations for $C$. For degrees 2, 3, and 4, such formulas are derived and presented in a systematic way in [21]. ${ }^{5}$ Weil [23] has traced the history back to Hermite. The formulas also appear in Duistermaat's book [24].

When $d=2$, we argue as follows. ${ }^{6}$ This time, let $\mathcal{M}$ be a line bundle on $C$ of degree 2. We choose a basis $u, v$ of the two-dimensional vector space $H^{0}(C, \mathcal{M})$, and then choose an element $w$ of $H^{0}\left(C, \mathcal{M}^{\otimes 2}\right)$ so that $u^{2}, u v, v^{2}, w$ is a basis of that space. Once again, we

\footnotetext{
${ }^{5}$ There is an "improved version" of these formulas in [22] which work well in characteristic 2 and 3.

${ }^{6}$ This argument is well-known in the mathematics literature, and was written out in appendix B of [25].
} 
enumerate the sections we know of various powers:

$$
\begin{aligned}
& H^{0}(C, \mathcal{M}) \quad \ni u, v \\
& H^{0}\left(C, \mathcal{M}^{\otimes 2}\right) \ni u^{2}, u v, v^{2}, w \\
& H^{0}\left(C, \mathcal{M}^{\otimes 3}\right) \ni u^{3}, u^{2} v, v v^{2}, v^{3}, u w, v w \\
& H^{0}\left(C, \mathcal{M}^{\otimes 4}\right) \ni u^{4}, u^{3} v, u^{2} v^{2}, u v^{3}, v^{4}, u^{2} w, u v w, v^{2} w, w^{2} .
\end{aligned}
$$

Since there are nine sections of $H^{0}\left(C, \mathcal{M}^{\otimes 4}\right)$, there must be one relation among them. Again, since there is no relation among sections of lower powers of $\mathcal{M}$, the coefficient of $w^{2}$ in the relation must be nonvanishing, and after rescaling we can assume that coefficient is 1. Thus, we get an equation of the form

$$
w^{2}+b_{0} u^{2} w+b_{1} u v w+b_{2} w^{2}=c_{0} u^{4}+c_{1} u^{3} v+c_{2} u^{2} v^{2}+c_{3} u v^{3}+c_{4} v^{4} .
$$

If the characteristic of $K$ is not 2, which we always assume in this paper, we can simplify this to an equation of the form

$$
w^{2}=e_{0} u^{4}+e_{1} u^{3} v+e_{2} u^{2} v^{2}+e_{3} u v^{3}+e_{4} v^{4} .
$$

by completing the square on $w$. This is standard form for degree two.

We will give a derivation of the formula for the Jacobian of $C$ in degree 2, based on some Galois theory which is often found in undergraduate abstract algebra classes. We start with a polynomial of degree 4 in a single variable, obtained from the right hand side of eq. (2.7) by setting $v=1$ :

$$
e_{0} u^{4}+e_{1} u^{3}+e_{2} u^{2}+e_{3} u+e_{4}=e_{0}\left(u^{4}+\frac{e_{1}}{e_{0}} u^{3}+\frac{e_{2}}{e_{0}} u^{2}+\frac{e_{3}}{e_{0}} u+\frac{e_{4}}{e_{0}}\right) .
$$

Galois theory constructs a finite extension $L$ of the field $K$ in which this polynomial has roots, and as is well-known (and visible in the classic formulas of Cardano), the first step is to define an associated polynomial of degree 3 . In fact, over the field $L$ the polynomial will factor as

$$
e_{0} \prod_{i=1}^{4}\left(u-r_{i}\right)
$$

and the cubic polynomial is related to this by forming the quantities

$$
s_{1}=r_{1} r_{2}+r_{3} r_{4}, \quad s_{2}=r_{1} r_{3}+r_{2} r_{4}, \quad s_{3}=r_{1} r_{4}+r_{2} r_{3},
$$

and using those to form the auxiliary cubic polynomial

$$
\prod_{j=1}^{3}\left(\widetilde{x}-s_{j}\right) .
$$

Note that

$$
\begin{aligned}
s_{1}-s_{2} & =\left(r_{1}-r_{4}\right)\left(r_{2}-r_{3}\right) \\
s_{1}-s_{3} & =\left(r_{1}-r_{3}\right)\left(r_{2}-r_{4}\right) \\
s_{2}-s_{3} & =\left(r_{1}-r_{2}\right)\left(r_{3}-r_{4}\right)
\end{aligned}
$$


which implies that the discriminants of the two polynomials are the same.

We can determine the equation of the auxiliary cubic polynomial by expressing the elementary symmetric functions of its roots in terms of the elementary symmetric functions $\sigma_{1}, \ldots, \sigma_{4}$ of $\left\{r_{1}, \ldots, r_{4}\right\}$. The calculation is straightforward, and the result is as follows:

$$
\begin{aligned}
s_{1}+s_{2}+s_{3} & =\sigma_{2} \\
s_{1} s_{2}+s_{1} s_{3}+s_{2} s_{3} & =\sigma_{1} \sigma_{3}-4 \sigma_{4} \\
s_{1} s_{2} s_{3} & =\sigma_{1}^{2} \sigma_{4}+\sigma_{3}^{2}-4 \sigma_{2} \sigma_{4}
\end{aligned}
$$

This implies that the cubic polynomial takes the form

$$
\widetilde{x}^{3}-\frac{e_{2}}{e_{0}} \widetilde{x}^{2}+\frac{e_{1} e_{3}-4 e_{0} e_{4}}{e_{0}^{2}} \widetilde{x}-\frac{e_{1}^{2} e_{4}+e_{0} e_{3}^{2}-4 e_{0} e_{2} e_{4}}{e_{0}^{3}} .
$$

If we rescale by substituting $x=e_{0} \widetilde{x}$ we find a cubic polynomial

$$
x^{3}-e_{2} x^{2}+\left(e_{1} e_{3}-4 e_{0} e_{4}\right) x-\left(e_{1}^{2} e_{4}+e_{0} e_{3}^{2}-4 e_{0} e_{2} e_{4}\right) .
$$

The corresponding homogenous equation

$$
y^{2}=x^{3}-e_{2} x^{2} z^{2}+\left(e_{1} e_{3}-4 e_{0} e_{4}\right) x z^{4}-\left(e_{1}^{2} e_{4}+e_{0} e_{3}^{2}-4 e_{0} e_{2} e_{4}\right) z^{6}
$$

is the equation of the Jacobian of the curve with equation (2.7). (To put it into Weierstrass form one should complete the cube in $x$.)

The Galois theory which goes along with this construction is based on the following exact sequence of groups:

$$
1 \rightarrow\left(\mathbb{Z}_{2}\right)^{2} \rightarrow \mathfrak{S}_{4} \rightarrow \mathfrak{S}_{3} \rightarrow 1
$$

As a consequence, if $L$ is the field in which the degree 4 polynomial (assumed to be general) has its roots so that the Galois group of $L$ over $K$ is the symmetric group $\mathfrak{S}_{4}$, there is an intermediate field $L^{\prime}$ whose Galois group over $K$ is $\mathfrak{S}_{3}$. The above construction explicitly builds the degree 3 polynomial whose roots lie in the field $L^{\prime}$.

Returning to the general problem of a curve of genus one over $K$ with a line bundle of degree $d$, if $d=3$ the story is straighforward: let $\mathcal{N}$ be a line bundle of degree 3 , and choose a basis $x, y, z$ of $H^{0}(C, \mathcal{N})$. There are 6 degree two monomials in $x, y$ and $z$ which matches the dimension of $H^{0}\left(C, \mathcal{N}^{\otimes 2}\right)$. However, since there are 10 degree three monomials in $x$, $y$, and $z$ but the space $H^{0}\left(C, \mathcal{N}^{\otimes 3}\right)$ has dimension 9 , there must be a relation of degree 3 . This expresses $C$ as a cubic curve in $\mathbb{P}_{K}^{2}$. The formula for the Weierstrass equation of the Jacobian is quite lengthy in this case, but can be found in [22].

If $d=4$ the story is also straightforward: let $\mathcal{P}$ be a line bundle of degree 4 , and choose a basis $x, y, z, t$ of $H^{0}(C, \mathcal{P})$. There are 10 degree 2 monomials in $x, y, z$, and $t$ yet the space $H^{0}\left(C, \mathcal{P}^{\otimes 2}\right)$ has dimension 8 , so there must be two relations of degree 2. Let $Q_{1}$, and $Q_{2}$ be a basis of the space of relations; then $C$ is described as a complete intersection $\left\{Q_{1}=Q_{2}=0\right\}$ in $\mathbb{P}_{K}^{4}$.

To describe the Weierstrass equation of the Jacobian in this case, we represent $Q_{1}$ and $Q_{2}$ as symmetric $4 \times 4$ matrices, and consider the determinant $\operatorname{det}\left(\lambda Q_{1}+\mu Q_{2}\right)$. This is a 
homogeneous polynomial of degree 4 in $\lambda$ and $\mu$, and thereby determines a curve $C^{\prime}$ of genus one and degree 2 as the double cover of $\mathbb{P}_{K}^{1}$ branched on the zeros of that homogeneous polynomial. The Jacobian of $C$ coincides with the Jacobian of $C^{\prime}$, and our previous formula for the degree 2 case provides the Weierstrass equation of the Jacobian, an explicit formula for which can be found in $[21,26,27]$. If the coordinates $x, y, z, t$ can be chosen such that one of the quadratics, say, $Q_{1}$, takes the special form

$$
Q_{1}=x y-z t,
$$

then $\left\{Q_{1}=0\right\}$ is itself a toric variety $\mathbb{P}_{K}^{1} \times \mathbb{P}_{K}^{1}$ and we again have a hypersurface in a toric variety.

The story above has recently been extended to $d=5$. There are 5 sections of the defining line bundle, and the equations can be presented as the vanishing of the $4 \times 4$ Pfaffians of a $5 \times 5$ matrix with entries that are linear functions of the 5 sections [28]. Formulas have been found for the Weierstrass equation of the Jacobian of the curve, and although the formulas are too large to write down as explicit polynomials, there is an algorithm for evaluating them [29].

\section{Singularities of Jacobian fibrations}

Dolgachev and Gross [9] have studied the Tate-Shafarevich group of a genus-one fibered threefold in considerable detail. Their analysis goes beyond the Calabi-Yau case and includes arbitrary genus-one fibered algebraic threefolds. The results fall short of giving an algorithm for computing the Tate-Shafarevich group, but they are strong enough to compute it in some important examples. The analysis is quite technical and we will not attempt to present the results here. However, many of the key features of their analysis are present in one particular example which we now describe following [9].

Let $f_{1}(\vec{x}), f_{2}(\vec{x}), f_{3}(\vec{x})$ be three general cubics in $\mathbb{P}^{2}$, and consider

$$
Y=\left\{(\vec{x}, \vec{u}) \in \mathbb{P}_{\vec{x}}^{2} \times \mathbb{P}_{\vec{u}}^{2} \mid u_{1} f_{1}(\vec{x})+u_{2} f_{2}(\vec{x})+u_{3} f_{3}(\vec{x})=0\right\} .
$$

We map $Y \rightarrow \mathbb{P}_{\vec{u}}^{2}$ by $(\vec{x}, \vec{u}) \mapsto \vec{u}$, and note that the fiber over $\vec{u}$ is a curve of genus one in $\mathbb{P}_{\vec{x}}^{2}$. This fibration has no section if the three cubics are general, but there is a 3 -section given by intersecting $Y$ with $x_{3}=0$, which gives three points in each fiber. (We stress that this example is not Calabi-Yau, but is nevertheless fibered by curves of genus one.)

The discriminant locus for $Y \rightarrow \mathbb{P}_{\vec{u}}^{2}$ can be computed with some methods from classical algebraic geometry. The computation was made in [30], and the result states that the discriminant is an irreducible curve in $\mathbb{P}_{\vec{u}}^{2}$ of degree 12 with 24 cusps and 21 nodes. Moreover, the total space $Y$ of this family is nonsingular, and the map $Y \rightarrow \mathbb{P}_{\vec{u}}^{2}$ is flat; this enables Dolgachev and Gross to identify the Tate-Shafarevich group in this case with a subgroup of $\mathbb{Z} / 3 \mathbb{Z}$, using their general results about Tate-Shafarevich groups for flat fibrations with nonsingular total space. Since $Y \rightarrow \mathbb{P}_{\vec{u}}^{2}$ itself does not have a section, the Tate-Shafarevich group must be nontrivial, so it must be $\mathbb{Z} / 3 \mathbb{Z}$.

A key result of [9] is that the Jacobian fibration $J(Y) \rightarrow \mathbb{P}_{\vec{u}}^{2}$ has the same discriminant locus as that of $Y \rightarrow \mathbb{P}_{\vec{u}}^{2}$. Thus, the Jacobian fibration in this example has a Weierstrass 
model whose discriminant is a curve of degree 12 with 24 cusps and 21 nodes. Now over a general point of the discriminant locus in a Weierstrass fibration, the fiber acquires a node (Kodaira type $I_{1}$ ) but the total space is smooth. Similarly, at a cusp in the discrimiant of a Weiestrass model, the fiber has a cusp (Kodaira type $I I$ ) but the total space is smooth. The only place where the total space of the Weierstrass model is singular is at the 21 nodes of the discriminant locus, where the Kodaira type of the fiber is $I_{2}$. The singularity in the total space is an ordinary quadratic singularity, otherwise known as a "conifold" singularity. ${ }^{7}$

In order to produce a nonsingular model of $J(Y) \rightarrow \mathbb{P}_{\vec{u}}^{2}$, one would like to find a "small resolution" of the conifold singularity. The standard way to do this would exploit the local factored form of the discriminant: the singularity can locally be written in the form $x y=h\left(u_{1}, u_{2}\right)$ in the affine chart $u_{3}=1$ with $h\left(u_{1}, u_{2}\right)$ representing the discriminant, and taking a local factorization $h=h_{1} h_{2}$ (where $h_{j}=0$ defines one of the local branches of the curve at its node, for $j=1,2)$, the blowup of $\left\{x=h_{1}=0\right\}$ produces the small resolution. Such a small resolution, if it existed, would give a flat family $\widetilde{J(Y)} \rightarrow \mathbb{P}_{\vec{u}}^{2}$ with nonsingular total space. However, since $h\left(u_{1}, u_{2}\right)$ is globally an irreducible curve, this factorization cannot be performed globally and the small resolution may not exist as an algebraic variety. ${ }^{8}$ The failure of small resolutions to exist globally has shown up a number of times in the past in applications to string theory, including [32], where it was responsible for a Stückelberg mechanism in effective theories of D-branes at singularities.

In fact, as shown in [9], there is no small resolution of the conifold singularities in this example. If there were, then the general results of [9] would imply that the Tate-Shafarevich group of $J(Y)$ is a subgroup of $\mathbb{Z} / 1 \mathbb{Z}$, i.e., it would have to be the trivial group. ${ }^{9}$ But since the Tate-Shafarevich group is actually $\mathbb{Z} / 3 \mathbb{Z}$, the small resolution cannot exist.

Notice that it is always possible to blowup the conifold point itself, giving a "big" resolution. However, any holomorphic three-form on $J(Y)$ which is nonvanishing near the conifold point necessarily has zeros along the exceptional divisor of the big resolution. Therefore, the big resolution is not suitable for studying Calabi-Yau threefolds. It also fails to have a flat fibration.

The conclusion that the Jacobian of a nonsingular genus-one fibration has conifold singularities (or possibly worse singularities) which cannot be resolved to give a flat family appears to be a general one, borne out by additional examples in [9] as well as further examples in this paper. The general theory of minimal models in the birational geometry of threefolds (see [33], for example) identifies these singularities as "Q- -factorial terminal singularities."

In addition to conifold singularities which cannot be resolved, Dolgachev and Gross find another geometric feature of $J(Y)$ in this example which (as we will see) helps to identify the physics of the corresponding M-theory compactification. Let us blow up the base at the nodes of the discriminant, and then blowup the singular locus of the total space,

\footnotetext{
${ }^{7}$ Note that all of these statements could be explicitly verified using the techniques of section 2 .

${ }^{8}$ The fact that "collisions" between Kodaira fibers of type $I_{2 k+1}$ and $I_{2 \ell+1}$ may obstruct the existence of flat families was first observed by Miranda [31].

${ }^{9}$ According to [9], the Tate-Shafarevich group for a flat family with nonsingular total space is an extension of a certain geometric group by $\mathbb{Z} / \delta \mathbb{Z}$, where $\delta$ is the minimum intersection number of a fiber with a multisection. For $Y, \delta=3$, but for $J(Y), \delta=1$.
} 
to obtain a new surface $\widehat{\mathbb{P}^{2}}$ with an elliptic fibration $\widehat{J(Y)} \rightarrow \widehat{\mathbb{P}^{2}}$ which is flat and has a nonsingular total space $\widehat{J(Y)}$. The Tate-Shafarevich group of $\widehat{J(Y)}$ is again $\mathbb{Z} / 3 \mathbb{Z}$, and this time can be identified with the torsion in cohomology, i.e., with ${ }^{10} H^{3}(\widehat{J(Y)}, \mathbb{Z})_{\text {tors }}$. Note that since the torsion is a birational invariant, this can also be seen as a torsion cohomology class on the "big blowup" of the conifold points on $J(Y)$.

As observed in [10] and studied further in [11], there are M-theory compactifications on certain singular spaces (spaces with "frozen" singularities) which are well-defined in spite of the singularities; the interpretation is that the M-theory 3-form has a discrete flux which obstructs the resolution of the singularities. We find ourselves in a similar situation here, with an M-theory model on the singular space $J(Y)$ which (due to having an F-theory limit in common with M-theory on $Y$ ) should be well-defined. Moreover, the space has a natural torsion 3 -form. ${ }^{11}$

This, then is our interpretation: the Jacobian of a genus-one fibered Calabi-Yau threefold $Y$ is an elliptic fibration $J(Y)$ with $\mathbb{Q}$-factorial terminal singularities which is equipped with an appropriate torsion class in such a way that M-theory compactified on $J(Y)$ with torsion flux for the M-theory 3-form is well-defined.

\section{Fiberwise duality with M-theory}

\subsection{Review of the F/M duality}

One way to define F-theory compactified on an elliptic fibration is to compactify M-theory on one of the cycles of the torus fiber down to IIA and then perform T-duality [37] to IIB. The T-dual circle decompactifies in the limit where the torus fiber shrinks to vanishing size, and in fact one obtains a IIB string theory compactified on the base of the elliptic fibration with varying axion-dilaton.

The argument goes as follows. Pick coordinates $z=x+\tau y$ with $0 \leq x, y \leq 1$ on the torus $E_{\tau}$. Then the Calabi-Yau metric on a $E_{\tau}$-fibration $Y$ over $B$ is presumably of the form $[38,39]$

$$
d s_{Y}^{2}=d s_{B}^{2}+\frac{v}{\tau_{2}} d z d \bar{z}+O\left(v^{2}\right)
$$

in the limit where the fiber volume $v \rightarrow 0$. The complex structure $\tau=\tau_{1}+i \tau_{2}$ of the fiber $E_{\tau}$ varies as a function of the base. One recognizes this as the M-theory lift, on the $x$-circle, of the IIA metric

$$
\begin{gathered}
d s_{M}^{2}=e^{4 \phi_{\mathrm{IIA}} / 3}\left(d x+C_{1}\right)^{2}+e^{-2 \phi_{\mathrm{IIA}} / 3} d s_{I I A}^{2}, \\
C_{\mathrm{IIA}}^{(1)}=\tau_{1} d y, \quad e^{4 \phi_{\mathrm{IIA}} / 3}=\frac{v}{\tau_{2}}, \quad d s_{\mathrm{IIA}}^{2}=\sqrt{\frac{v}{\tau_{2}}}\left(v \tau_{2} d y^{2}+d s_{B}^{2}\right) .
\end{gathered}
$$

\footnotetext{
${ }^{10}$ This identification proceeds $[9,17]$ via the cohomological Brauer group $B r^{\prime}(\widehat{J(Y)})=H^{2}\left(\widehat{J(Y)}, \mathcal{O} \frac{\times}{J(Y)}\right)$, which coincides with the torsion in cohomology since $\widehat{J(Y)}$ is nonsingular [34], a result which holds since $\widehat{J(Y)}$ has no holomorphic 1 -forms or 2-forms.

${ }^{11}$ There is a subtlety here, in that the torsion 3-form exists not on $J(Y)$ itself but on a blowup. However, as shown in [35], in the context of explaining some examples of discrete torsion in type IIA string theory [36], a torsion class on a blowup of $J(Y)$ can lead to corresponding physical effects on $J(Y)$ itself. The torsion in [35] was related to the cohomological Brauer group in that case just as it is in the present case.
} 
To dualize to type IIB, we now perform T-duality on the remaining $y$-circle. Its circumference, in the IIA metric, is $L_{y}=v^{3 / 4} \tau_{2}^{1 / 4}$. Hence, T-duality amounts to rescaling

$$
T: y \mapsto v^{-3 / 2} \tau_{2}^{-1 / 2} y
$$

which results in the IIB metric

$$
d s_{\mathrm{IIB}, S}^{2}=\sqrt{\frac{v}{\tau_{2}}}\left(\frac{d y^{2}}{v^{2}}+d s_{B}^{2}\right) .
$$

Moreover, T-duality shifts the dilaton $\phi$ and identifies corresponding RR-forms with one leg in the circle direction, namely

$$
e^{\phi_{\mathrm{IIB}}}=\frac{1}{L_{y}} e^{\phi_{\mathrm{IIA}}}=\frac{1}{\tau_{2}}, \quad C_{\mathrm{IIB}}^{(0)} d y=C_{\mathrm{IIA}}^{(1)} .
$$

Finally, we rescale the IIB metric from the string frame to the Einstein frame to obtain

$$
d s_{\mathrm{IIB}, E}^{2}=e^{-\frac{\phi_{\mathrm{IIB}}}{2}} d s_{\mathrm{IIB}, S}^{2}=\sqrt{v}\left(\frac{d y^{2}}{v^{2}}+d s_{B}^{2}\right) .
$$

Remarkably, the $\tau$-dependence of the metric has completely disappeared. The fiber volume $v$ is constant over the base, so the metric is just a metric on $[0,1] \times B$ and decompactifies to $\mathbb{R} \times B$ in the limit $v \rightarrow 0$. The entire $\tau$-dependence is in the IIB axion-dilaton, which is simply $C_{\mathrm{IIB}}^{(0)}+i e^{-\phi_{\mathrm{IIB}}}=\tau_{1}+i \tau_{2}=\tau$

\subsection{Fiberwise duality}

The argument presented above is clearly naïve: the fiber complex structure $\tau$ varies holomorphically, so if it is non-constant then it must have zeros and poles where the ansatz eq. (4.1) cannot be valid. Even worse, there are $\mathrm{SL}(2, \mathbb{Z})$ transformations along loops which encircle the discriminant locus $\Delta \subset B$, so there is no global choice of $x$ and $y$-circle.

Hence, we also need to appeal to fiberwise duality to complete the M-theory/IIB duality. Locally, over the base $B$, there is no preferred $\operatorname{SL}(2, \mathbb{Z})$ frame. But that choice also has no physical significance: possible Dehn twists on the F-theory elliptic curve just correspond to the changing S-duality frame of the IIB axion-dilaton. We can apply the above duality on sufficiently small open sets and glue the IIB description via $\mathrm{SL}(2, \mathbb{Z})$ transformations. The result is IIB on the base with varying axion-dilaton $\tau$. The actual value of $\tau$ is not uniquely defined, but it is unique up to $\mathrm{SL}(2, \mathbb{Z})$-transformations. The representation $\pi_{1}(B-\Delta) \rightarrow \mathrm{SL}(2, \mathbb{Z})$ is part of the defining data of the elliptic fibration.

However, $\mathrm{SL}(2, \mathbb{Z})$-transformations are not the entire symmetry group by which one can glue local patches. In addition, there are translations along the fiber. This obviously does not preserve the zero-section (i.e., the locus of points serving as "0" in the group structure on each fiber), so the ensuing fibration will, in general, only be a genus-one fibration. At first sight, allowing translations seems to be very boring: $\tau$ does not change if we translate along the torus, so no physical quantity appears to know about it. However, this argument really only tells us that no field knows about the translations locally, which is tautologically true, as the geometry has local sections. But global monodromies can and will depend on this additional freedom, and in section 7.5 we will see an explicit example. 


\subsection{Tate-Shafarevich group}

To be able to act requires an identity element on the part of the actor, but not on the part of the acted upon. A vector space acts on an affine space by translations. Or, relevant for our purposes, an elliptic curve acts on a curve of genus one with the same $\tau$ by translations. In particular, recall from section 2 that for every genus-one fibration $Y$ there is an elliptic fibration $J(Y)$. Sections of the Jacobian act by translation on the genus-one fibration, turning the Mordell-Weil group of the Jacobian into a subgroup of the birational symmetries of $Y$.

To construct new genus-one fibrations, we can start with an elliptic fibration $Y \rightarrow B$ and choose translations locally in each patch of the base $B$. Gluing together the patches by the translations creates a genus-one fibration which may or may not have sections. Explicitly, let $\mathcal{A}$ be the sheaf of rational sections of the elliptic fibration. By definition, $\mathcal{A}$ is a sheaf of Abelian groups with respect to fiber-wise addition, which we write as "+". We are interested in a collection $\sigma_{\alpha \beta} \in \mathcal{A}\left(U_{\alpha} \cap U_{\beta}\right)$ of local sections that fits together on triple overlaps, that is, $\sigma_{\alpha \beta}+\sigma_{\beta \gamma}=\sigma_{\alpha \gamma}$. Moreover, changing the local sections by a coboundary is just a reparametrization and yields the same genus-one fibration after gluing. Therefore, the distinct genus-one fibrations that can be constructed by twisting the elliptic fibration are in one-to-one correspondence with the elements of the cohomology group

$$
\amalg_{B}(\mathcal{A})=H^{1}(B, \mathcal{A}),
$$

also known as the Tate-Shafarevich group. By the above discussion, we can identify its elements with the set of genus-one fibrations having the same Jacobian, that is, having the same $\tau$.

This construction works as stated whenever the fibration $Y \rightarrow B$ is generic, i.e., has only Kodaira fibers of types $I_{0}, I_{1}$ and $I I$ (and no non-Abelian gauge symmetry). For more complicated fibrations, the sheaf $\mathcal{A}$ misses too much of the structure of $Y$, and the more detailed analysis of [9] must be used.

\subsection{Relation with discrete torsion}

On an elliptic curve, that is, a curve of genus one with marked point $\sigma$, a choice of point $p$ amounts to a choice of line bundle $\mathcal{O}(p-\sigma)$ with vanishing first Chern class. Hence, we could use the defining data of the Tate-Shafarevich group just as well to glue something by tensoring with a line bundle of vanishing first Chern class. Except for the "vanishing $c_{1}$ " part, one recognizes this as the familiar gerbe data defining the twist of a projective vector bundle. For torsion gerbe characteristic classes, this is also knows as discrete torsion in string theory. In more fancy language, we can think of the sheaf $\mathcal{A}$ as the degree-zero part of the relative Picard sheaf

$$
0 \longrightarrow \mathcal{A} \longrightarrow \underline{P i c}(Y / B) \longrightarrow \mathbb{Z} \longrightarrow 0,
$$

except that the Picard sheaf may not be well-defined for sufficiently complicated fibrations, leading to considerable technical difficulties for which we refer the reader to $[9,16,17]$. Nevertheless, the induced map in cohomology

$$
\amalg_{B}(\mathcal{A})=H^{1}(B, \mathcal{A}) \longrightarrow H^{1}(B, \underline{\operatorname{Pic}}(Y / B))
$$


and the Leray spectral sequence

$$
\cdots \longrightarrow B r^{\prime}(B) \longrightarrow B r^{\prime}(Y) \longrightarrow H^{1}(B, \underline{\operatorname{Pic}}(Y / B)) \longrightarrow \cdots
$$

for $\mathcal{O}_{Y}^{\times}$on the projection $Y \rightarrow B$ links the Tate-Shafarevich group to the (cohomological) Brauer group and gerbes (for generic fibrations).

However, note that only the Brauer group on the total space modulo the pull-back of the Brauer group on the base has a chance of contributing according to eq. (4.10). That is, the Tate-Shafarevich group provides similar but strictly independent global information from B-fields and gerbes in Type IIB. In the special case where $\operatorname{dim} B=1$ or where all fibers are irreducible (hence no non-Abelian gauge symmetry), the relationship between the Brauer and the Tate-Shafarevich groups becomes particularly simple. In this case [9] the quotient of the Brauer groups is indeed the only contribution,

$$
\amalg_{B}(\mathcal{A})=\operatorname{coker}\left(B r^{\prime}(B) \rightarrow B r^{\prime}(Y)\right) .
$$

Hence, under these simplifying assumptions the IIB gerbes on the base and the TateShafarevich group of the fibration combine together into the Brauer group of the total space of the fibration.

The IIB fluxes should, more precisely, be thought of as classes in a suitable version of K-theory. For example, for orientifolds the correct flavor of K-theory is KR-theory, Real equivariant with respect to the orientifold involution. It would be nice to understand this better and have a direct connection to the Tate-Shafarevich group that does not proceed via cohomology.

\section{Moduli of genus-one fibrations}

\subsection{Weierstrass hypersurface}

So far we have argued that the degrees of freedom in F-theory include the Tate-Shafarevich group, elements of which correspond geometrically to distinct genus-one fibrations with the same Jacobian fibration. Allowing genus-one fibrations has direct physical consequences. In this section, we will see that it corrects the uncharged hypermultiplet count. In the next section, we will find new ways to break gauge symmetry. However, before getting ahead of ourselves, let us start with proper elliptic fibrations giving rise to SU(5) gauge theory to make contact with physics literature. Only later, starting with section 5.6, will we consider genus-one fibrations that do not admit a section. In order to make use of the strong anomaly cancellation conditions, let us focus on the case of genus-one fibered Calabi-Yau threefolds compactifying F-theory down to 6 dimensions.

The simplest way to construct an elliptic fibration over a fixed base $B$ is as a Weierstrass hypersurface. That is, consider the $\mathbb{P}^{2}[2,3,1]$ bundle

$$
X=\mathbb{P}\left(\mathcal{O}_{B}(-2 K) \oplus \mathcal{O}_{B}(-3 K) \oplus \mathcal{O}_{B}\right)[2,3,1] .
$$

For suitable bases $B$, an anticanonical hypersurface $Y \subset X$ is a Calabi-Yau threefold with only canonical singularities for which there exists a smooth Calabi-Yau resolution $\widetilde{Y} \rightarrow Y$. 
In order to avoid tensionless strings in our models, we are only interested hypersurfaces whose resolution has a fibration is flat, i.e., has only one-dimensional fibers. If the base is a toric variety with fan $\Sigma_{B}$, then the ambient space $X=X_{\Sigma}$ is also a toric variety. To construct the polytope for $X$, we note that the anticanonical hypersurface equation takes the long Weierstrass form

$$
y^{2}=x^{3}+a_{1} x y z+a_{2} x^{2} z^{2}+a_{3} y z^{3}+a_{4} x z^{4}+a_{6} z^{6}
$$

with coefficients $a_{i} \in \Gamma\left(\mathcal{O}_{B}\left(K^{-i}\right)\right)$. The hypersurface equation defines its Newton polytope and we define $\Sigma$ as its normal fan, that is, the face fan of the dual polytope. ${ }^{12}$ For example, if $B=\mathbb{P}^{2}$ then one obtains the weighted projective space $X=\mathbb{P}^{4}[1,1,1,6,9]$ as ambient fourfold. The defining polytope is:

\begin{tabular}{|c|c|c|ccc|ccc|}
\hline$u$ & $v$ & $w$ & $x$ & $y$ & $z$ & & & \\
\hline 9 & 0 & 0 & 0 & -1 & 3 & 2 & 1 & 1 \\
6 & 0 & 0 & -1 & 0 & 2 & 1 & 1 & 0 \\
-1 & 1 & 0 & 0 & 0 & 0 & 0 & 0 & 0 \\
-1 & 0 & 1 & 0 & 0 & 0 & 0 & 0 & 0 \\
\hline \multicolumn{2}{|l|}{ three tops $^{13}$} & \multicolumn{2}{|c|}{ fiber } & \multicolumn{2}{c|}{$\begin{array}{c}\text { facet } \\
\text { interior }\end{array}$} \\
\hline
\end{tabular}

The toric variety $X$ corresponding to eq. (5.1) inherits singularities from the fiber $\mathbb{P}^{2}[2,3,1]$. However, for $B=\mathbb{P}^{2}$ there is a unique toric resolution preserving the fibration, so we will not dwell on this issue.

The choice of ambient space induces additional structure on the hypersurface beyond that of a generic torus-fibered Calabi-Yau threefold over $B$. In particular,

- The toric divisor $V(z) \cap Y$ is a section of $Y$, namely

$$
s: B \rightarrow Y, \quad b \mapsto(b,[1: 1: 0])
$$

- The self-intersection of the canonical class on the toric fiber $\mathbb{P}^{2}[2,3,1]$ equals 6 . Therefore, $-K_{X} \cap Y$ is a family of 6 -sections. It can be chosen to contain a section as an irreducible component, which we can subtract to leave us with a 5-section. The particular choice

$$
\left\{y^{2}=x^{3}\right\} \cap Y=\left\{z\left(a_{1} x y+a_{2} x^{2} z+a_{3} y z^{2}+a_{4} x z^{3}+a_{6} z^{5}\right)=0\right\} \cap Y
$$

of 5-section in this family is called "the Tate divisor" in the physics literature [40].

\footnotetext{
${ }^{12}$ Depending on the base $B$, the dual need not be a lattice polytope. That is, the Newton polytope need not be reflexive. This means that the singular variety does not admit a resolution to a smooth Calabi-Yau hypersurface.

${ }^{13}$ See section 5.2 for the definition of "top". The above example has only trivial (consisting of a single point) tops, which would not illustrate the definition adequately.
} 

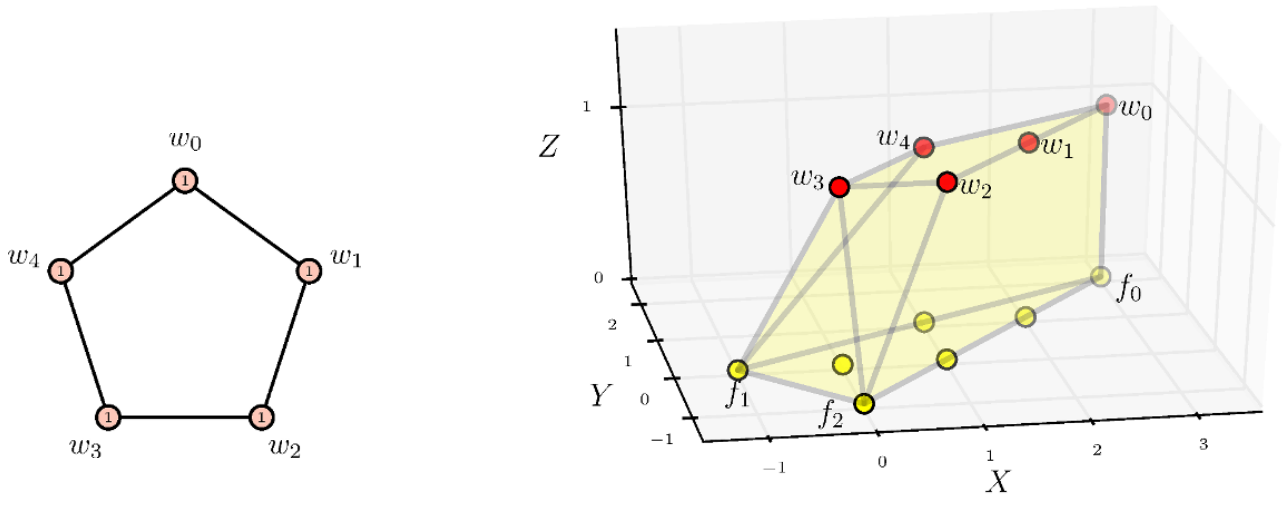

Figure 1. The split $\mathrm{SU}(5)$ top and associated extended Dynkin diagram, the $I_{5}$ Kodaira fiber.

A generic Calabi-Yau hypersurface in the projective space bundle $X$ has a nonsingular total space and only three types of Kodaira fibers: $I_{0}$ (a nonsingular fiber), $I_{1}$ (a fiber with a node), and $I I$ (a fiber with a cusp). In particular, its F-theory compactification has no gauge symmetry at all, only $h^{2,1}(Y)+1=273$ uncharged hypermultiplets. In order to generate non-Abelian interactions, one needs to find special loci in the complex structure moduli space where more complicated Kodaira fibers appear. At the same time, one has to ensure that there still exists a flat resolution. Examples of terminal singularities as well as examples of canonical singularities whose resolution is not flat exist. While not insurmountable obstacles, these are very real complications.

\subsection{Elliptic fibration with a toric SU(5)}

Instead of trying to specialize the Weierstrass equation by hand, we can also specialize the ambient space in a way that enforces particular Kodaira fibers. In particular, this avoids potential terminal singularities: a generic three-dimensional Calabi-Yau hypersurface in a toric variety can always be resolved into a smooth threefold [41].

For example, consider the following split $I_{5}$ toric elliptic fibration over $\mathbb{P}^{2}$ [42-44] with Hodge numbers $h^{1,1}=6, h^{2,1}=171$. The ambient space is the toric variety with polytope $\nabla$ whose points are

\begin{tabular}{|c|c|c|c|c|c|c|c|c|c|c|c|}
\hline$u$ & & $w_{0}$ & $w_{1}$ & $w_{2}$ & $w_{3}$ & $w_{4}$ & & $f_{1}$ & $f_{2}$ & & \\
\hline 6 & 0 & 3 & 2 & 1 & 0 & 1 & 3 & 0 & -1 & 11 & 2 \\
\hline 4 & 0 & 2 & 1 & 0 & 0 & 1 & 2 & -1 & 0 & 01 & 1 \\
\hline-1 & & 1 & 1 & 1 & 1 & 1 & 0 & 0 & 0 & 00 & 0 \\
\hline-1 & & 0 & 0 & 0 & 0 & 0 & 0 & 0 & 0 & 00 & 0 \\
\hline \multicolumn{7}{|c|}{ three tops } & \multicolumn{3}{|c|}{ fiber } & \multicolumn{2}{|c|}{ facet interior } \\
\hline
\end{tabular}

and which is fibered over $\mathbb{P}^{2}$ via the projection to the last two coordinates. In terms of homogeneous coordinates, this is the map

$$
\pi: X_{\nabla} \rightarrow \mathbb{P}^{2}, \quad\left[u: v: w_{0}: \cdots: w_{4}: f_{0}: f_{1}: f_{2}\right] \mapsto\left[u: v: w_{0} w_{1} w_{2} w_{3} w_{4}\right]
$$


The Dynkin diagram of the gauge group can be seen from the tops [45-51] of the fibration. We recall their definition and important properties:

- Given a ray $\rho$ of the base fan, the preimage $\pi^{-1}(\rho) \subset \nabla$ is called a "top".

- Regardless of the dimension of the codimension-two fibered toric variety, a top is always a 3-dimensional lattice polytope with the origin on one facet (namely the fiber polygon).

- The top over $\rho$ defines the gauge group over the discriminant component $V(\rho)$ as follows:

- The edge graph (with corresponding integral points) not meeting the fiber polygon equals the quotient of the Dynkin diagram by the monodromy.

- The Dynkin labels are the height of the points over the fiber polygon.

- The toric (multi-)sections correspond to the vertices of the fiber polygon, and their intersection with the fiber irreducible components are also visible as the edges of the top.

In the case of an elliptically fibered $\mathrm{K} 3$, there are only two rays in the base $\mathbb{P}^{1}$ and they divide the K3 polytope in a top and a bottom half. This is the origin of the name, but in general there is one top for each ray in the base.

In our example there are three tops over the three rays of the fan of the base $\mathbb{P}^{2}$. Two of them are trivial with only a single vertex added over the fiber polygon. The third accounts for the $\mathrm{SU}(5)$ gauge group and is shown in figure 1. Using toric geometry to translate the polyhedral data into an algebraic variety $[42,52,53]$, each integral point of the $w$-top corresponds to an irreducible component of the (complex two-dimensional) toric fiber over $w=0$ in the base. The hypersurface equation cuts out $\mathbb{P}^{1}$ 's in each irreducible toric fiber component. In the case at hand, the hypersurface equation yields a single $\mathbb{P}^{1}$ in each of the five components, linked as in the $I_{5}$ Kodaira fiber.

\subsection{Adding a single $\mathrm{U}(1)$}

For physics applications it is desirable to have a non-trivial Mordell-Weil group, unlike the threefold constructed in section 5.2. The torsion part of the Mordell-Weil group is a discrete symmetry of the low energy effective action, and the rank equals the number of $U(1)$ factors in the gauge group. Both have important phenomenological applications, for example ruling out certain operators that would lead to excessive proton decay. Although not necessary for the remainder of this paper, we now make a small digression to discuss ways to realize additional U(1) factors. The reader not interested in these can skip ahead to section 5.6.

The most straightforward idea to generate a $U(1)$ is to take the Weierstrass hypersurface and restrict the complex structure further until an extra section appears. (In fact, as argued in [25], this is the only way to do it.) If one wants the additional U(1) to be toric, then this means one should find a point to add to the fiber polytope such that it induces a new toric section and such that the convex hull is again reflexive. It is easy to see that adding $(-1,-1,0,0)$ to the polytope in eq. (5.6) works, and the new polytope is drawn 

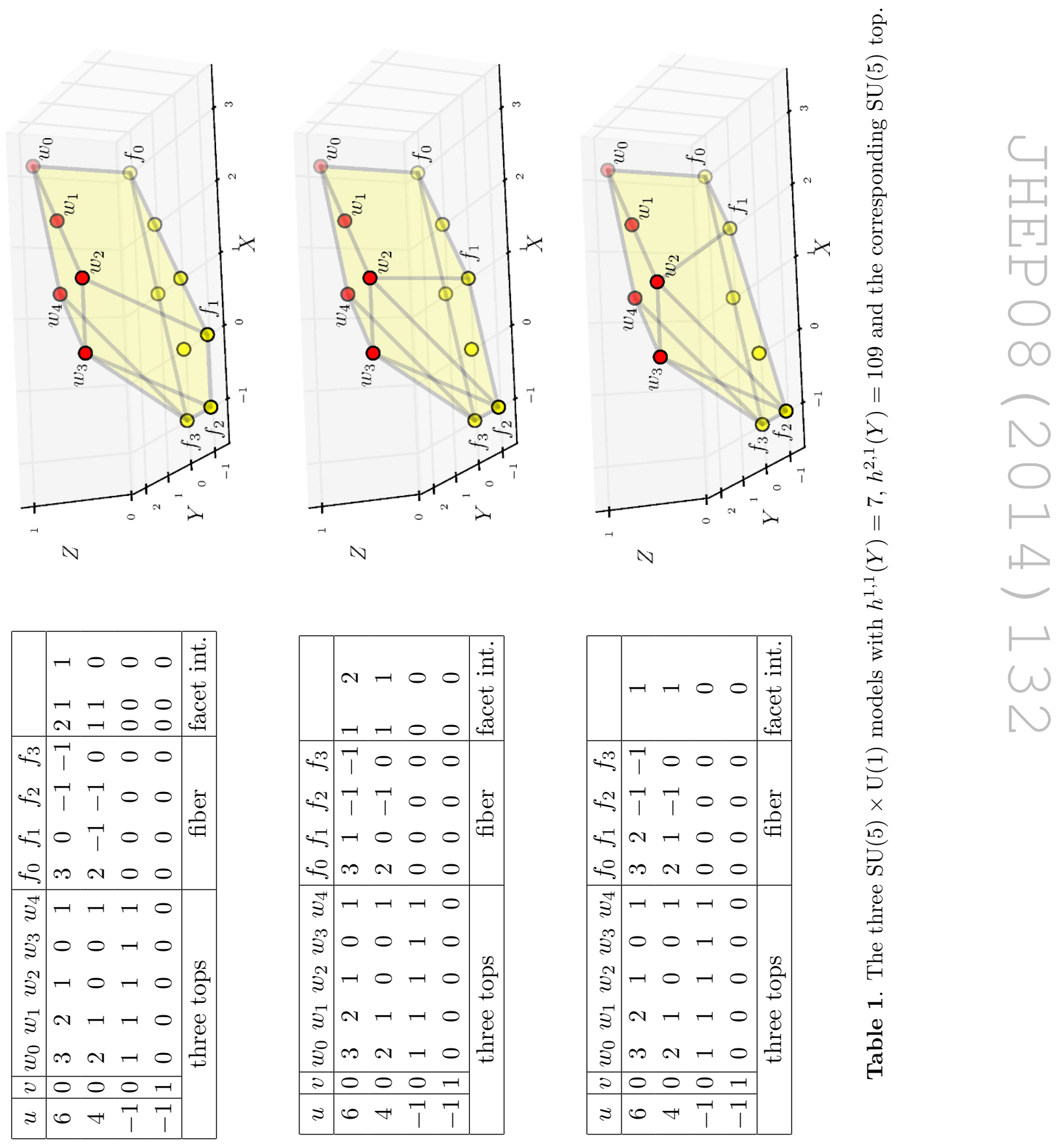

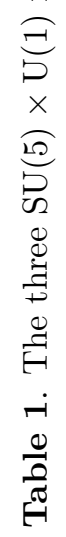


in the top row of table 1. In fact, the new Calabi-Yau hypersurface has three sections: $V\left(f_{0}\right), V\left(f_{1}\right)$, and $V\left(f_{2}\right)$. The new $h^{1,1}(Y)=7$, so the additional toric sections generate a rank-one Mordell-Weil group. Therefore, the gauge group is enhanced to $\mathrm{SU}(5) \times \mathrm{U}(1)$. The self-intersection number of the canonical class of the generic toric fiber drops from 6 on $\mathbb{P}^{2}[2,3,1]$ to 5 . Hence the Tate divisor degenerated into a 4 -section and the new section. This construction is known as the "U(1)-restricted Tate model" in the physics literature.

This is not the only way to generate a single $\mathrm{U}(1)$ using toric techniques, ${ }^{14}$ and in table 1 we list two more polytopes that give rise to the same spectrum. In fact, it is easy to see that the top two cases contain the bottom polytope, that is, the hypersurfaces of the top two toric varieties are special limits of the hypersurface of the bottom toric variety. In particular, we recognize that the extra $\mathrm{U}(1)$ comes from the additional vertex of the fiber polytope. This extra vertex yields an additional integral point of the $\nabla$-polytope not in the interior of a facet, and therefore increases $h^{1,1}$ by one. Hence, the reason for the additional $\mathrm{U}(1)$ is this additional vertex in the toric picture, and not the fact that one of the three is the restriction of the Weierstrass hypersurface to a special point. That the three polytopes are contained in each other implies that the Weierstrass model is the same, but the details of the resolution to a smooth threefold are different. In particular, the toric description of the codimension-two degeneration where the $\underline{\mathbf{5}}_{3}$ matter is localized differs.

- In the first $\mathrm{SU}(5) \times \mathrm{U}(1)$ polytope of table 1 we recognize a conifold singularity defined by the square $f_{1}, f_{2}, w_{3}, w_{2}$. In constructing a smooth Calabi-Yau threefold, one must resolve it in one of two ways. Either way, this extra curve is the additional $\mathbb{P}^{1}$ in 7 out of the 19 fibers where the $I_{5}$ Kodaira fiber degenerates into an $I_{6}$ Kodaira fiber. Depending on which resolution one takes, this curve is contained in the $V\left(f_{1}\right) \cap Y$ or the $V\left(f_{2}\right) \cap Y$ section.

- In the second and third $\mathrm{SU}(5) \times \mathrm{U}(1)$ polytope of table 1 , none of the extra $\mathbb{P}^{1}$ in the codimension-two $I_{6}$ fibers are toric curves in the top. All toric sections intersect the irreducible components of $I_{6}$ codimension-two fibers transversely.

None of these differences in resolution impact the physics of the $\mathrm{SU}(5) \times \mathrm{U}(1)$ F-theory model.

\section{$5.4 \mathrm{U}(1)$ charge assignments}

In the case at hand all charged hypermultiplets arise from isolated curves. Their U(1) charge of the hypermultiplet on a curve $C$ under the $\mathrm{U}(1)$ of a section $S \in M W(Y)$ is determined by the intersection

$$
C \cdot \pi(S)=C \cdot S+\sum_{i, j}\left(S \cdot \theta_{i}\right)\left(A^{-1}\right)_{i j}\left(\Theta_{j} \cdot C\right)
$$

where the $\theta_{i}$ are the curves carrying the roots, that is, the irreducible components of the gauge group discriminant component not intersecting the given section, the $\Theta_{j}$ are the divi-

\footnotetext{
${ }^{14} \mathrm{~A}$ systematic analysis of the toric ambient spaces for elliptic curves which can be employed to construct multiple toric sections for elliptically-fibered varieties was given in [44, 54-57].
} 

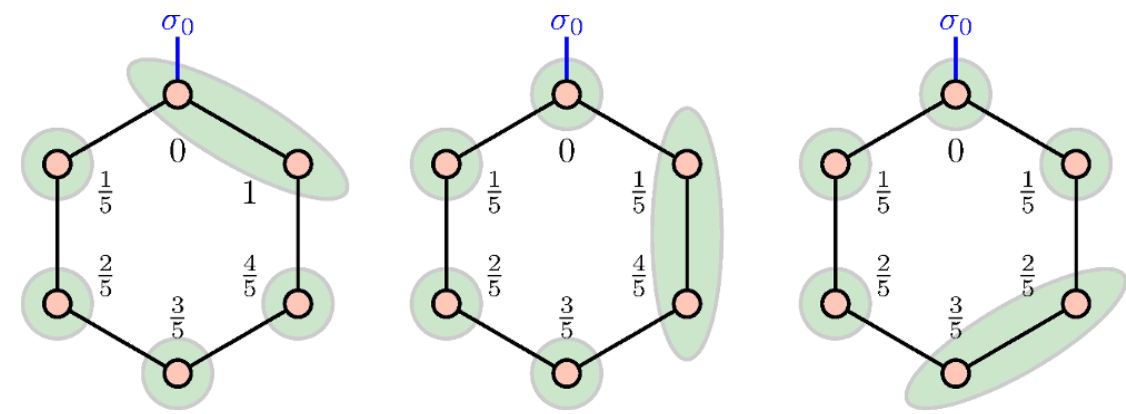

Figure 2. The $\mathrm{U}(1)$ charge of a $\underline{\mathbf{5}}$ hypermultiplet localized at a codimension-two fiber where the $I_{5}$ discriminant (green) degenerates to $I_{6}$ (red). Pick the red node where the section generating the Mordell-Weil group intersects the fiber. The number next to the node is the ratio of the U(1) charge relative to a $\underline{\mathbf{1}}$ hypermultiplet.

sors swept out by the curve $\theta_{i}$ over the discriminant, and $A$ is the Cartan matrix of the gauge group. For example, for the $\underline{\mathbf{5}}$ hypermultiplets the U(1) charge works out as in figure $2 .{ }^{15}$

In addition to the $\mathrm{SU}(5)$-charged hypermultiplets, there are also a number of hypermultiplets charged only under the U(1) whose curves are localized away from the non-Abelian discriminant component. That is, they are localized at special points of the $I_{1}$ discriminant component. We will only encounter the three simplest cases in the following discussion:

- Smooth point: $u=v$ or $u=v^{2}$

(Milnor number 0)

- Node: $u v=0$

(Milnor number 1)

- Cusp: $u^{2}=v^{3}$

(Milnor number 2)

It is of practical importance to be able to determine the number of such singularities in the $I_{1}$ component efficiently for a generic hypersurface. One useful trick is to take the polynomial $\delta_{1}(u, v, w)$ whose vanishing defines $I_{1}$ and compute its discriminant with respect to one of the variables. This then factorizes into linear, quadratic, and cubic factors that can be counted easily. For example, the $\mathrm{SU}(5)$ model has 171 cusps in the $I_{1}$ discriminant component and no nodes. In fact, without any section there cannot be any node for intersection-theoretic reasons: the resolved Calabi-Yau would have an $I_{2}$ Kodaira fiber over the node, but without a section there is no divisor available to be the Poincaré dual of the irreducible fiber component not intersecting the given section.

The three $\mathrm{SU}(5) \times \mathrm{U}(1)$ models with $h^{1,1}=7, h^{2,1}=109$, see table 1 , have 171 cusps and 63 nodes in the $I_{1}$ discriminant component. Computing the intersections with the toric sections, one finds the $\mathrm{U}(1)$ charges of the hypermultiplets to be

$$
7 \times \underline{\mathbf{5}}_{3} \oplus 12 \times \underline{\mathbf{5}}_{2} \oplus 3 \times \underline{\mathbf{1 0}}_{1} \oplus 63 \times \underline{\mathbf{1}}_{5}
$$

which satisfies the U(1) anomaly cancellation conditions for an appropriate choice of GreenSchwarz term. For completeness, let us recall the anomaly cancellation conditions for a

\footnotetext{
${ }^{15}$ Note that we will always normalize $\mathrm{U}(1)$ charges to be integral. The electron charge is -3 .
} 
single non-Abelian gauge group $G$ and a single $\mathrm{U}(1)$ when the base $B$ is $\mathbb{P}^{2}$. A GreenSchwarz term proportional to

$$
\int-\frac{3}{2} \operatorname{tr} R^{2}+2 b \operatorname{tr}_{G} F_{G}^{2}+2 \tilde{b} F_{\mathrm{U}(1)}^{2}
$$

will cancel the anomalies provided that $[25,58]$ :

$$
\begin{array}{rlrl}
18 b=\sum_{i} A_{R_{i}}-A_{\mathrm{ad}}, & 0=\sum_{i} B_{R_{i}}-B_{\mathrm{ad}}, \quad 3 b^{2}=\sum_{i} C_{R_{i}}-C_{\mathrm{ad}}, \\
0 & =\sum_{i} E_{R_{i}} r_{i}, & 18 \tilde{b} & =\sum_{i} \operatorname{dim}\left(R_{i}\right) r_{i}^{2}, \\
b \tilde{b} & =\sum_{i} A_{R_{i}} r_{i}^{2}, & 3 \tilde{b}^{2} & =\sum_{i} \operatorname{dim}\left(R_{i}\right) r_{i}^{4} .
\end{array}
$$

where the model has hypermultiplets transforming in the representations $\left(R_{i}, r_{i}\right)$. In the example at hand, all anomalies cancel ${ }^{16}$ for $b=1$ and $\tilde{b}=120$.

\subsection{Three U(1)'s}

In order to realize three toric $\mathrm{U}(1)$ factors in a toric hypersurface, the generic ambient space fiber must be $d P_{6}[44,51,57]$. One possibility for $\mathrm{SU}(5) \times \mathrm{U}(1)^{3}$ gauge symmetry with $h^{1,1}=9, h^{2,1}=52$ is:

\begin{tabular}{|c|c|ccccc|cccccc|}
\hline$u$ & $v$ & $w_{0}$ & $w_{1}$ & $w_{2}$ & $w_{3}$ & $w_{4}$ & $f_{0}$ & $f_{1}$ & $f_{2}$ & $f_{3}$ & $f_{4}$ & $f_{5}$ \\
\hline 3 & 0 & 0 & -1 & -1 & 0 & 0 & 1 & 1 & 0 & -1 & -1 & 0 \\
1 & 0 & -1 & -1 & 0 & 1 & 0 & 1 & 0 & -1 & -1 & 0 & 1 \\
-1 & 0 & 1 & 1 & 1 & 1 & 1 & 0 & 0 & 0 & 0 & 0 & 0 \\
-1 & 1 & 0 & 0 & 0 & 0 & 0 & 0 & 0 & 0 & 0 & 0 & 0 \\
\hline \multicolumn{8}{c|}{ three tops } & \multicolumn{7}{c|}{ fiber } \\
\hline
\end{tabular}

All SU(5) models studied so far have 19 points on the discriminant where the vanishing degree jumps from $(0,0,5)$ to $(0,0,6)$ and three points where it jumps to $(2,3,7)$. Therefore, the $\mathrm{SU}(5)$-charged hypermultiplets are $19 \times \underline{\mathbf{5}} \oplus 3 \times \underline{\mathbf{1 0}}$ which is precisely what is needed to cancel the non-Abelian gauge anomaly

$$
18 b=19 \cdot 1+3 \cdot 3-10, \quad 0=19 \cdot 1+3 \cdot(-3)-10, \quad 3 b^{2}=19 \cdot 0+3 \cdot 3-6 .
$$

The $\mathrm{SU}(5) \times \mathrm{U}(1)^{3}$ model with toric sections, see eq. (5.12), has 171 cusps and 122 nodes.

\subsection{No section}

After the detour on F-theory models with a section, we now finally present an example without a section. In addition to the SU(5) model with a toric section, see eq. (5.6), there are 15 further fibered toric hypersurfaces with an $I_{5}$ discriminant component and $h^{1,1}=6$, that is, no $\mathrm{U}(1)$. They do differ in the number of complex structure parameters, ranging from 90 to 49 instead of $h^{2,1}=171$ of eq. (5.6). This is quite a common phenomenon. In

\footnotetext{
${ }^{16}$ The non-Abelian anomaly coefficients, see section $\mathrm{A}$, are $A_{\underline{\mathbf{1}}}=B_{\underline{\mathbf{1}}}=C_{\underline{\mathbf{1}}}=0, A_{\underline{\mathbf{5}}}=B_{\underline{\mathbf{5}}}=1, C_{\underline{\mathbf{5}}}=0$, $A_{\underline{10}}=-B_{\underline{10}}=C_{\underline{10}}=3$, and $A_{\mathrm{ad}}=B_{\mathrm{ad}}=10, C_{\mathrm{ad}}=6$.
} 


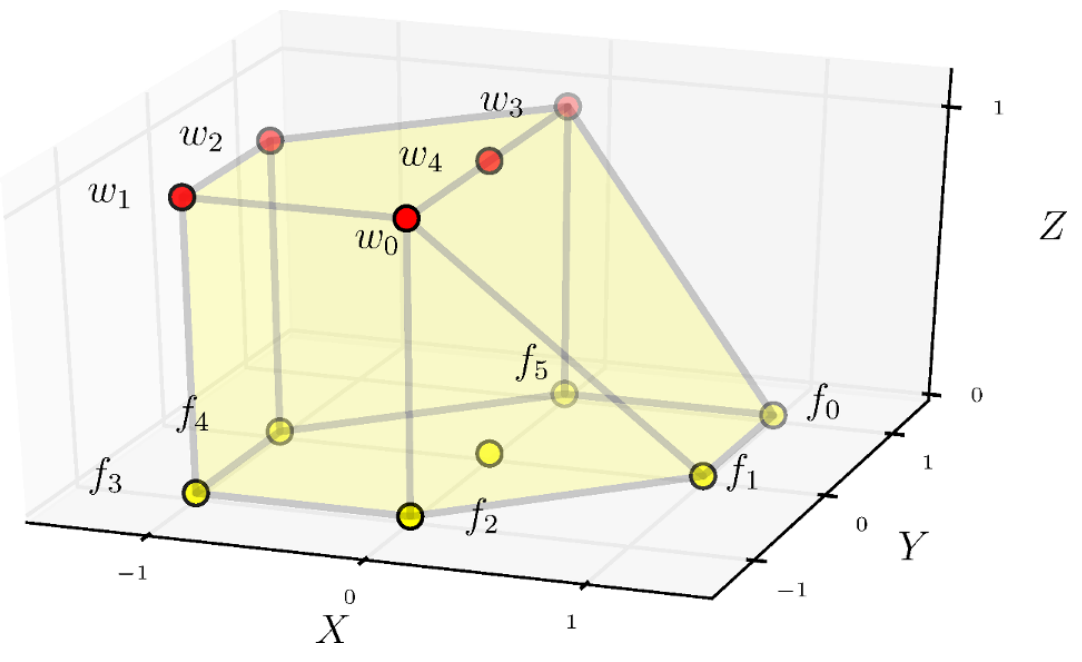

Figure 3. The $\mathrm{SU}(5) \times \mathrm{U}(1)^{3}$ top.

table 2 we list all toric hypersurfaces fibered over $\mathbb{P}^{2}$ such that the gauge group is only $\mathrm{SU}(n)$ and the non-Abelian discriminant component is a toric curve in the base $\mathbb{P}^{2}$. Just to be explicit, we will use the $\mathrm{SU}(5)$ model with $h^{2,1}=90$ in the remainder of this section as an example. The polytope of the ambient toric variety is

\begin{tabular}{|c|c|ccccc|ccc|c|}
\hline$u$ & $v$ & $w_{0}$ & $w_{1}$ & $w_{2}$ & $w_{3}$ & $w_{4}$ & $f_{0}$ & $f_{1}$ & $f_{2}$ & \\
\hline 2 & 0 & 1 & 1 & 0 & -1 & 0 & 1 & 1 & -1 & 1 \\
1 & 0 & 1 & 0 & 0 & 1 & 1 & 1 & -1 & 0 & 0 \\
-1 & 0 & 1 & 1 & 1 & 1 & 1 & 0 & 0 & 0 & 0 \\
-1 & 1 & 0 & 0 & 0 & 0 & 0 & 0 & 0 & 0 & 0 \\
\hline \multicolumn{7}{c|}{ three tops } & \multicolumn{3}{|c|}{ fiber } & facet int. \\
\hline
\end{tabular}

with the fibration being defined by the projection on the last two coordinates. The fiber polygon is that of $\mathbb{P}^{2}[1,1,2]$ which does not lead to a toric section, see figure 4 . In fact, there is no section at all: by a direct computation, the $I_{1}$ discriminant component of a generic hypersurface can be seen to have 171 cusps and 81 nodes. As we mentioned earlier, the presence of a node means that a single section cannot serve as Poincaré dual divisor to both irreducible components of the $I_{2}$ resolution. Hence there cannot be a section. However, a two-section is clearly allowed since it can easily intersect both components of the $I_{2}$. And, in fact, the $\mathbb{P}^{2}[1,1,2]$ fiber polytope does induce 2 toric two-sections, $V\left(f_{0}\right) \cap Y$ and $V\left(f_{1}\right) \cap Y$. Each of them meets the $I_{5}$ Kodaira fiber of the non-Abelian discriminant component in two distinct components.

The Calabi-Yau threefold $Y$ is a genus-one fibration without a section and therefore not an elliptic fibration. However, it has a Jacobian fibration $J(Y)$ and $J(Y)$ in turn has a Weierstrass model $W_{Y}=\left\{y^{2}=x^{3}+f x+g\right\}$. (A Weierstrass model always has a section by construction.) The Weierstrass model also has 81 terminal singularities over the 81 


\begin{tabular}{|c|ccc|}
\hline$G$ & $h^{1,1}$ & $h_{\text {expected }}^{2,1}$ & $h^{2,1}$ \\
\hline $\mathrm{SU}(2)$ & 3 & 231 & $\left\{231,123,119,111^{2}, 107,81\right.$, \\
& & & $77,76,75,73,72,71\}$ \\
$\mathrm{SU}(3)$ & 4 & 208 & $\{208,110,104,100,98$, \\
& & & $\left.73,71,68,65,64^{4}, 61\right\}$ \\
$\mathrm{SU}(4)$ & 5 & 189 & $\left\{189,99,98,93,90,89,87^{2}, 83\right.$, \\
& & & $\left.66,62,60,59^{3}, 58,57^{2}, 56,55\right\}$ \\
$\mathrm{SU}(5)$ & 6 & 171 & $\{171,90,87,84,83,81,80,78$, \\
& & & $\left.57,54^{2}, 53,52,51^{2}, 50,49\right\}$ \\
$\mathrm{SU}(6)$ & 7 & 154 & $\left\{154,151,79^{2}, 77,74,73,71^{3}\right.$, \\
& & & $\left.68^{2}, 64,47^{4}, 46^{2}, 43^{2}, 40\right\}$ \\
$\mathrm{SU}(7)$ & 8 & 138 & $\left\{138,68,66^{2}, 62,60,42,41,38\right\}$ \\
$\mathrm{SU}(8)$ & 9 & 123 & $\left\{123,60,59,54,51^{2}, 47,36\right\}$ \\
$\mathrm{SU}(9)$ & 10 & 109 & $\{109,53,46,44\}$ \\
$\mathrm{SU}(10)$ & 11 & 96 & $\{96,47,39,32\}$ \\
$\mathrm{SU}(11)$ & 12 & 84 & $\{84,30\}$ \\
$\mathrm{SU}(12)$ & 13 & 73 & $\{73\}$ \\
$\mathrm{SU}(13)$ & 14 & 63 & $\{63\}$ \\
$\mathrm{SU}(14)$ & 15 & 54 & $\{54\}$ \\
$\mathrm{SU}(15)$ & 16 & 46 & $\{46\}$ \\
$\mathrm{SU}(16)$ & 17 & 39 & $\{39\}$ \\
$\mathrm{SU}(17)$ & 18 & 33 & $\{19\}$ \\
$\mathrm{SU}(24)$ & 25 & 19 & \\
\hline
\end{tabular}

Table 2. Hodge numbers of the Calabi-Yau threefolds in toric varieties, fibered over $\mathbb{P}^{2}$, with gauge group a pure $\mathrm{SU}(n)$ such that the non-Abelian discriminant component is a toric curve. The expected number of parameters $h_{\text {expected }}^{2,1}=271-23 n+\frac{n(n+1)}{2}$. Exponents indicate that the value of $h^{2,1}$ is realized by multiple polytopes. Only the hypersurfaces with $h_{\text {expected }}^{2,1}$ complex structure moduli are actual elliptic fibrations, others are genus-one fibrations without section.

nodes in the $I_{1}$ discriminant component, so it is too singular to define a string theory or M-theory compactification. However, as described in section 3, these terminal singularities come along with a discrete torsion class in the cohomology of the big blowup, and the Mtheory vacuum with 3 -form flux on that class is expected to be a sensible M-theory model with 81 "frozen" singularities.

To further verify that compactifying F-theory on a genus-one fibration without section makes sense, we now turn to the anomaly cancellation conditions which provide a very stringent consistency check.

\section{Gravitational anomaly cancellation}

Naïvely, the genus-one fibrations are at odds with the gravitational anomaly cancellation [13]. With fixed gauge group and changed matter content, the number of uncharged 


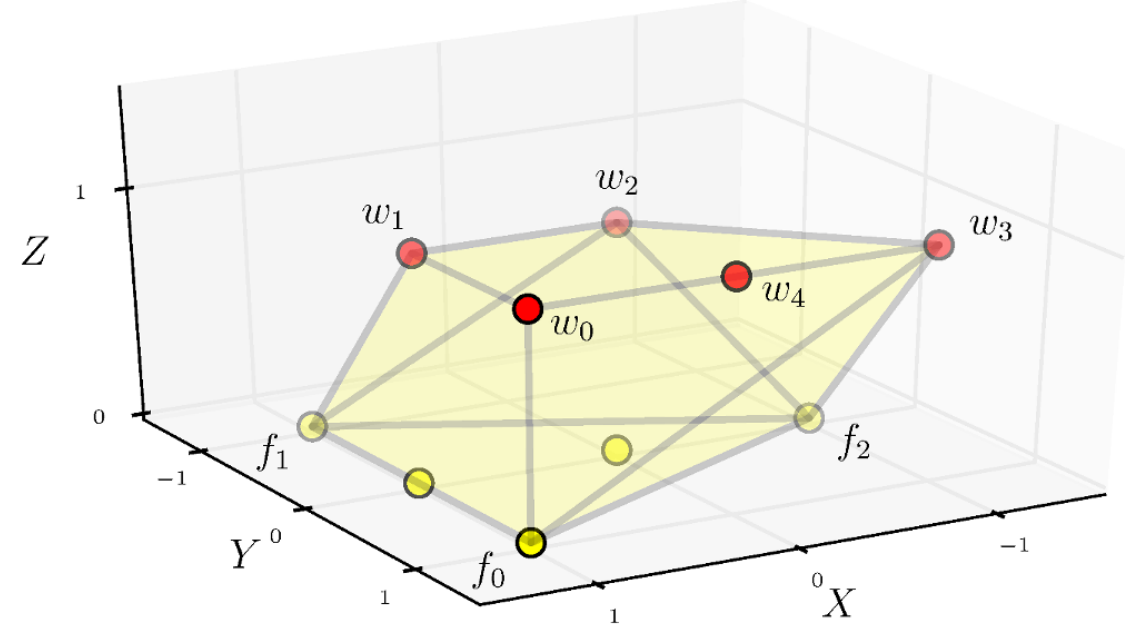

Figure 4. The SU(5) top without section.

hypermultiplets must satisfy $H_{u}+H_{c}-V+29 T=273$. The standard lore is that the uncharged hypermultiplets are the $h^{2,1}(Y)$ complex structure moduli plus one universal hypermultiplet, for a total of $H_{u}=h^{2,1}(Y)+1$. This cancels the anomaly for an elliptic fibration as it is related to its Euler number [59, 60]. A genus-one fibration has fewer complex structure moduli and, at least naively, not enough uncharged hypermultiplets. In fact, the missing complex structure moduli are easily understood from the nodes in the $I_{1}$ discriminant component: the complex structure moduli determine the position of the $I_{1}$, and each node imposes one additional constraint. For example, the genus-one fibration from section 5.6 has $h^{2,1}(Y)=90$ and 81 nodes in the $I_{1}$. If we add those integers, we arrive at 171 which is equal to the number of complex structure moduli of the SU(5) elliptic fibration eq. (5.6). This suggests that the uncharged hypermultiplet count for genus-one fibrations is corrected to

$$
H_{u}=h^{2,1}(Y)+1+\#\{\text { nodes }\} .
$$

In fact, Witten's quantization argument for rigid curves [61] tells us that there is an additional hypermultiplet localized at the $I_{2}$ fiber over the node, so this correction is to be expected. What is new is that this localized hypermultiplet is uncharged. It is instructive to compare this hypermultiplet with a geometrically similar hypermultiplet that arises [25] when there are two sections of the fibration (instead of a single 2-section). On the righthand side of figure 5, we have drawn the 2-section and how it intersects the $I_{2}$ fiber over the node in the base. Locally, this is indistinguishable from two distinct sections generating a rank-one Mordell-Weil group (left figure). The difference is only visible globally: either the two local sections stay separate globally or they meet and are exchanged at a ramification point. In either case there is a massless hypermultiplet, either charged under the $\mathrm{U}(1)$ determined by the difference of the sections, or uncharged in the case of a 2 -section. 

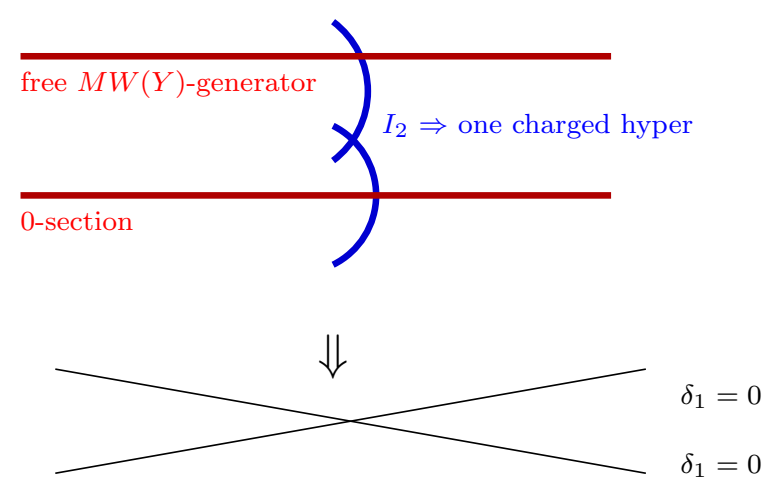
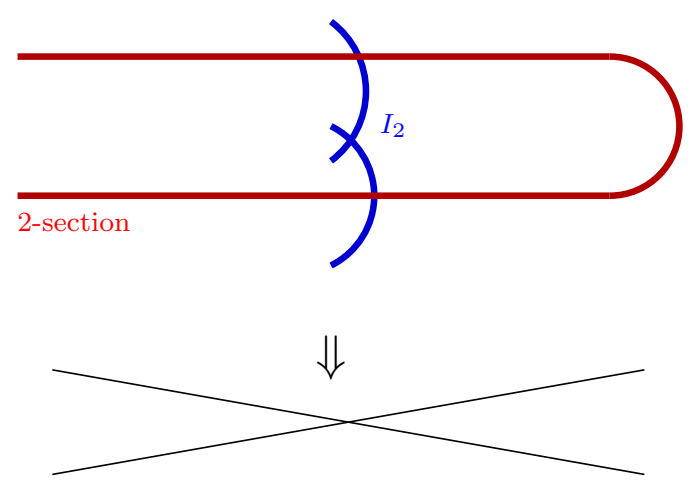

Figure 5. Left: a localized hypermultiplet charged under a U(1) from the difference of two sections. Right: a global monodromy preventing the $\mathrm{U}(1)$ gauge charge, resulting in a localized uncharged hypermultiplet.

Note that the U(1) charge of a hypermultiplet changes sign if we exchange the role of the zero-section and the generating section of the Mordell-Weil group. Therefore, we can no longer assign $\mathrm{U}(1)$ charges to the localized hypermultiplets if the global monodromy breaks the gauge group. ${ }^{17}$

\section{$7 \quad$ Triality of $E_{6}$ and monodromies}

\subsection{Unbroken gauge group}

In this section we will take a closer look at fibrations with a Kodaira fiber of type $I V^{*}$, which translates into an $E_{6}$ gauge group possibly broken by monodromies. In fact, we will find new monodromy effects in section 7.5. But for the sake of a coherent presentation we will first review the two known classes, which are known as split and non-split case [63].

To start, let us look at the unique split $E_{6}$ toric elliptic fibration over $\mathbb{P}^{2}$, that is, the model whose gauge group is unbroken $E_{6}$ and nothing else. The ambient space is the toric variety with polytope $\nabla_{1}$ whose points are

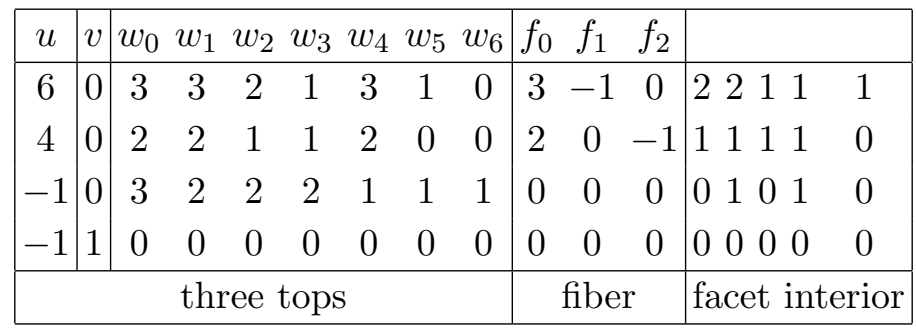

and which is fibered over $\mathbb{P}^{2}$ via the projection to the last two coordinates. In terms of homogeneous coordinates, this is the map

$$
\pi: X_{\nabla_{1}} \rightarrow \mathbb{P}^{2}, \quad\left[u: v: w_{0}: \cdots: w_{6}: f_{0}: f_{1}: f_{2}\right] \mapsto\left[u: v: w_{0}^{3} w_{1}^{2} w_{2}^{2} w_{3}^{2} w_{4} w_{5} w_{6}\right]
$$

In the case at hand, the $w$-top and Dynkin diagram are shown in figure 6 , which confirms that the gauge group is $E_{6}$ with no monodromy. Since the fiber polygon is that of

\footnotetext{
${ }^{17}$ Similar issues are discussed in [62].
} 

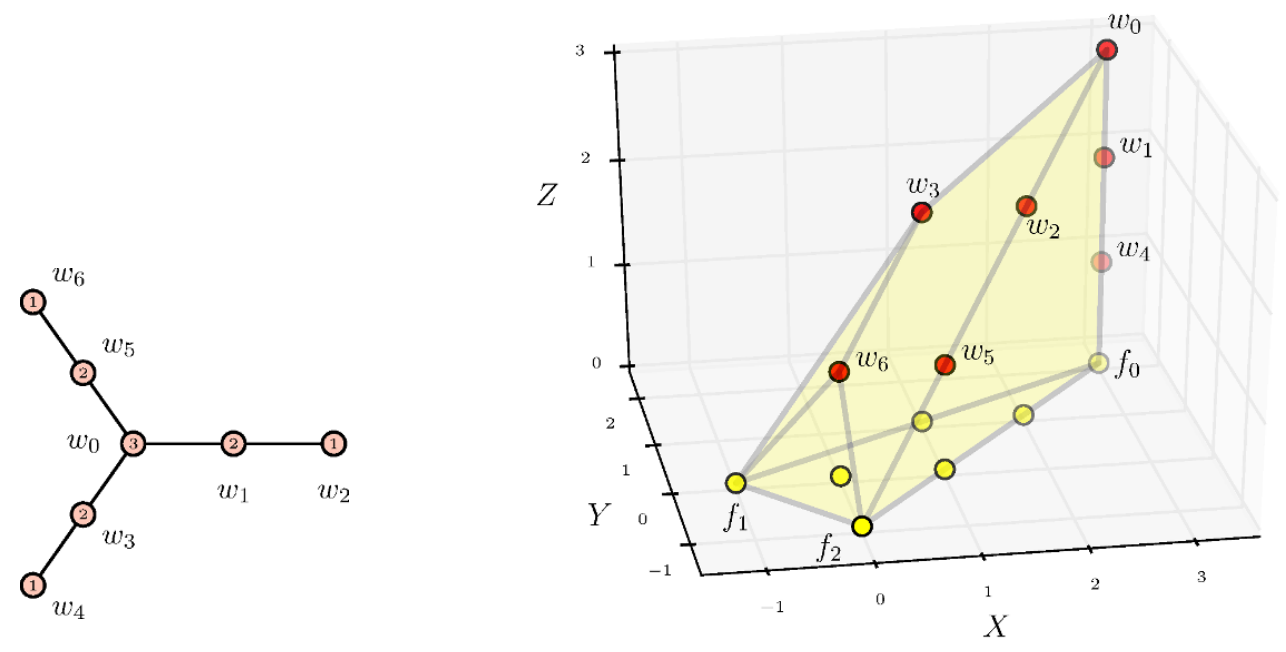

Figure 6. The split $E_{6}$ top and associated Dynkin diagram.

$\mathbb{P}^{2}[1,2,3]$, we are in the favorable case where a Calabi-Yau hypersurface coincides with its Weierstrass model. There is a toric section $V\left(f_{0}\right)$ meeting the fiber component $V\left(w_{4}\right) \cap F$ in a point [44], a toric 2-section $V\left(f_{2}\right)$ meeting $V\left(w_{5}\right) \cap F$ and $V\left(w_{6}\right) \cap F$ in one point each, and a toric 3-section $V\left(f_{3}\right)$ meeting $V\left(w_{6}\right) \cap F$ in a single point and meeting $V\left(w_{3}\right) \cap F$ in a point with multiplicity two (for a total of 3 points). It is straightforward to check that the Tate algorithm [63-65] and monodromy equation [60] agrees with the above identification of the gauge group.

The elliptic fiber degenerates further over a number of points in the base. The most basic degeneration is where the vanishing degree $(\operatorname{deg} f, \operatorname{deg} g, \operatorname{deg} \Delta)$ of the Weierstrass coefficients and discriminant is enhanced from $(3,4,8)$ to $(3,5,9)$. In general the degeneration encodes charged matter content of the 6-d theory, and this particular one yields a $\underline{\mathbf{2 7}}$ of $E_{6}$, and requires an intersection of the $I V^{*}$ discriminant component and the $I_{1}$ discriminant component with multiplicity 4 . Hence, by degree counting, one expects that the toric $I V^{*}$ discriminant component intersect the $I_{1}$ discriminant component in 7 points of multiplicity 4. A numerical analysis of a general toric hypersurface equation confirms this. To summarize, the generic Calabi-Yau hypersurface $Y_{1} \subset X_{\nabla_{1}}$ has Hodge numbers $h^{1,1}\left(Y_{1}\right)=8, h^{2,1}\left(Y_{1}\right)=161$. The 6 -d F-theory compactification is a $E_{6}$ gauge theory with $7 \times \underline{\mathbf{2 7}}$. This satisfies the gauge and gravitational anomaly cancellation conditions

$$
\begin{gathered}
H_{u}=h^{2,1}+1=162, \quad H_{c}=7 \cdot 27, \quad V=78 \\
H_{u}+H_{c}-V=273, \quad 18 b=7 \cdot 6-24, \quad 3 b^{2}=7 \cdot 3-18
\end{gathered}
$$

for $b=1$.

\subsection{Alternative gauge groups}

Strictly speaking, the smooth Calabi-Yau threefold constructed in section 7.1 does not yield any gauge interactions. Instead, one must contract some irreducible fiber components. The 
ensuing singularity is what is responsible for the 5-d gauge group, from which we infer the 6-d gauge group. (The compactification to 5 -d gives the Coulomb branch of the 6 - $\mathrm{d}$ theory, which may exhibit a variety of unbroken subgroups, all containing the Cartan subgroup.) The conventional way in which the 6 -d group was determined was to generate a 5 -d gauge group by contracting all irreducible fiber components except the one meeting the section, which produced a maximal group. The inference that this must coincide with the 6 -d group is clear.

Note that when there is more than one section a choice must be made, but the groups obtained are all isomorphic to each other. However, when there is no section, the situation is different and it is possible to produce different maximal 5-d gauge groups which are not contained in one another (somewhat analogous to the situation described in [66]). We will see explicit examples of this shortly.

However, let us first just consider the $E_{6}$ theory from section 7.1. We can, for example, shrink all components except the three irreducible fiber components at the three ends of the $E_{6}$ extended Dynkin diagram, even though the unique section passes through only one of them. This amounts to the Levi type ${ }^{18}$ branching rule

$$
\begin{aligned}
E_{6} & \supset D_{4}, \\
\operatorname{ad}\left(E_{6}\right) & =\operatorname{ad}\left(D_{4}\right) \oplus 2\left(\underline{\boldsymbol{s}}_{v} \oplus \underline{\mathbf{8}}_{s} \oplus \underline{\mathbf{8}}_{c}\right) \oplus 2 \times \underline{\mathbf{1}}, \\
\underline{\mathbf{2 7}} & =\underline{\mathbf{8}}_{v} \oplus \underline{\mathbf{8}}_{s} \oplus \underline{\mathbf{8}}_{c} \oplus 3 \times \underline{\mathbf{1}} .
\end{aligned}
$$

The anomaly virtual representation restricts to

$$
H-V=7 \times \underline{\mathbf{2 7}} \ominus \operatorname{ad}\left(E_{6}\right)=5\left(\underline{\mathbf{s}}_{v} \oplus \underline{\mathbf{8}}_{s} \oplus \underline{\mathbf{8}}_{c}\right) \oplus 19 \times \underline{\mathbf{1}} \ominus \operatorname{ad}\left(D_{4}\right),
$$

and we obtain a $D_{4}=\mathrm{SO}(8)$ gauge theory with 5 copies of vector, spinor, and conjugate spinor as well as $H_{u}=h^{2,1}+1+19$ uncharged hypers. Of course we recognize this as the Higgs mechanism: some of the vector multiplets got massive, eating a hypermultiplet partner in the process. In particular, the anomaly cancellation condition is preserved.

By contrast, let us now shrink all irreducible fiber components except the central one ${ }^{19}$ in the $E_{6}$ extended Dynkin diagram, see figure 6 . This is allowed by the geometry, that is, there exists a particular triangulation of the face fan of the polyhedron such that $V\left(w_{0}\right)$ is the only fibral divisor that does not vanish at a particular face of the Kähler cone. Contracting two simply laced nodes creates an $A_{2}$ singularity, so the resulting gauge group is $A_{2}^{3}=\mathrm{SU}(3)^{3}$. The corresponding branching rule is

$$
\begin{aligned}
E_{6} & \supset \mathrm{SU}(3)^{3}, \\
\operatorname{ad}\left(E_{6}\right) & =(\underline{\mathbf{8}}, \underline{\mathbf{1}}, \underline{\mathbf{1}}) \oplus(\underline{\mathbf{1}}, \underline{\mathbf{8}}, \underline{\mathbf{1}}) \oplus(\underline{\mathbf{1}}, \underline{\mathbf{1}}, \underline{\mathbf{8}}) \oplus(\underline{\mathbf{3}}, \underline{\mathbf{3}}, \underline{\mathbf{3}}) \oplus(\underline{\overline{\mathbf{3}}}, \underline{\overline{\mathbf{3}}}, \underline{\overline{\mathbf{3}}}), \\
\underline{\mathbf{2}} & =(\underline{\mathbf{3}}, \underline{\overline{\mathbf{3}}}, \underline{\mathbf{1}}) \oplus(\underline{\mathbf{1}}, \underline{\mathbf{3}}, \underline{\overline{\mathbf{3}}}) \oplus(\underline{\overline{\mathbf{3}}}, \underline{\mathbf{1}}, \underline{\mathbf{3}}) .
\end{aligned}
$$

This is an extended type branching rule, corresponding to the removal of a node from the extended Dynkin diagram. Note that the $E_{6}$ and $A_{2}^{3}$ theories stand on the same footing as

\footnotetext{
${ }^{18}$ That is, a branching rule corresponding to the removal of nodes from the (non-extended) Dynkin diagram.

${ }^{19}$ Note that a section cannot pass though the central node: its multiplicity is three, so only ( $\left.3 n\right)$-sections can pass through it.
} 

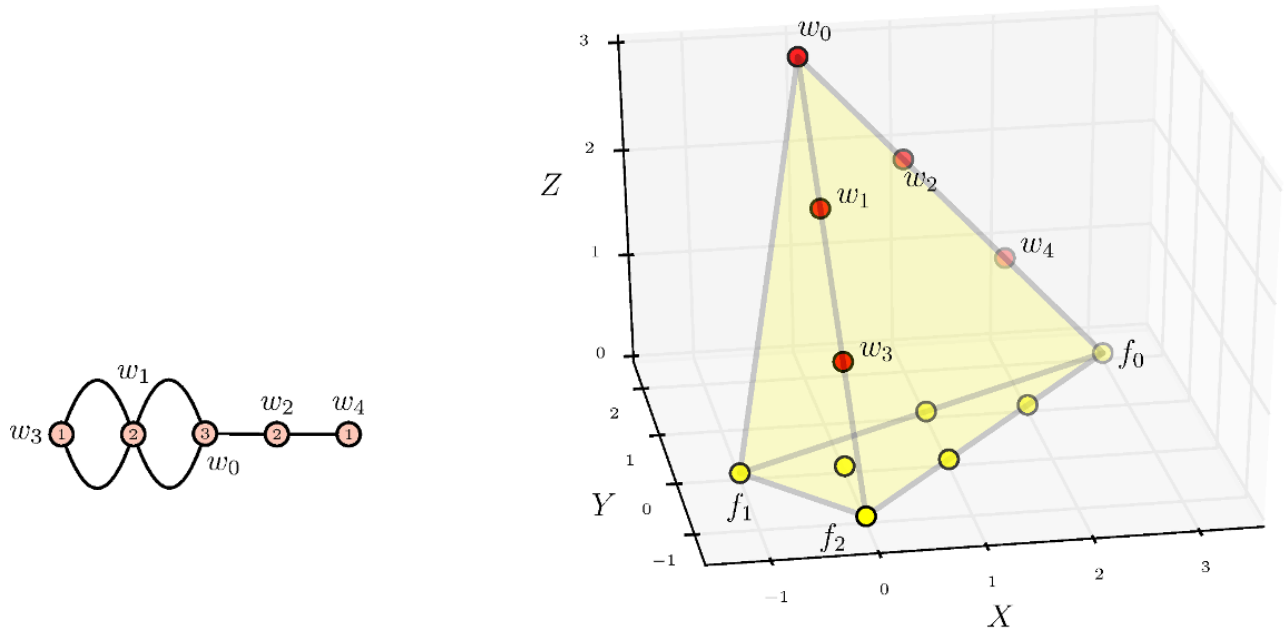

Figure 7. The $F_{4}$ top with $\mathbb{Z}_{2}$ monodromy and associated contracted Dynkin diagram.

both correspond to the removal of a single node from the extended Dynkin diagram. We are just picking a different compact subgroup of the affine $E_{6}$. In particular, neither can be obtained by the Higgs mechanism from the other. Note that the 6-d theory obtained this way is not a standard gauge theory as it contains exotic vector multiplets in the $(\underline{\mathbf{3}}, \underline{\mathbf{3}}, \underline{\mathbf{3}})$ and $(\underline{\overline{3}}, \underline{\overline{3}}, \underline{\overline{3}})$ representation in addition to the gauge multiplets.

\subsection{The non-split case}

We now proceed to the so-called non-split $I V^{*}$, that is, a $I V^{*}$ Kodaira fiber with a $\mathbb{Z}_{2}$ monodromy exchanging two of the three legs [67]. This is interpreted as $E_{6}$ broken to $F_{4}$ by the monodromy. The toric ambient space corresponds to the polytope $\nabla_{2}$ with points

\begin{tabular}{|c|c|ccccc|cccc|ccc|cc|}
\hline$u$ & $v$ & $w_{0}$ & $w_{1}$ & $w_{2}$ & $w_{3}$ & $w_{4}$ & $f_{0}$ & $f_{1}$ & $f_{2}$ & & & & & \\
\hline 0 & 7 & 0 & 0 & 1 & 0 & 2 & 3 & -1 & 0 & 0 & 1 & 1 & 1 & 2 \\
0 & 4 & 2 & 1 & 2 & 0 & 2 & 2 & 0 & -1 & 1 & 0 & 1 & 1 & 1 \\
-1 & 0 & 3 & 2 & 2 & 1 & 1 & 0 & 0 & 0 & 1 & 0 & 0 & 1 & 0 \\
-1 & 1 & 0 & 0 & 0 & 0 & 0 & 0 & 0 & 0 & 0 & 0 & 0 & 0 & 0 \\
\hline \multicolumn{7}{c|}{ three tops } & \multicolumn{1}{c|}{ fiber } & facet interior \\
\hline
\end{tabular}

The coordinates are again chosen such that the only non-trivial discriminant component comes from the $w$-top, which is depicted in figure 7 . The toric section $V\left(f_{0}\right)$ intersects the irreducible fiber component $V\left(w_{4}\right) \cap F$ in a single point, and the toric 3-section $V\left(f_{2}\right)$ intersects the irreducible fiber component $V\left(w_{0}\right) \cap F$ in a point with multiplicity 3 . The toric 2-section $V\left(f_{2}\right)$ intersects the fiber component $V\left(w_{3}\right) \cap F$ in two points. Since the latter has multiplicity one, the fiber component $V\left(w_{3}\right) \cap F$ must consist of two irreducible components. This explains why the Dynkin diagram is contracted as on the left hand side of figure 7 .

The standard choice in contracting the fiber components is to contract every one except $V\left(w_{4}\right)$. This leads to a $F_{4}$ gauge theory, namely $E_{6}$ broken by the $\mathbb{Z}_{2}$ monodromy. As for the 

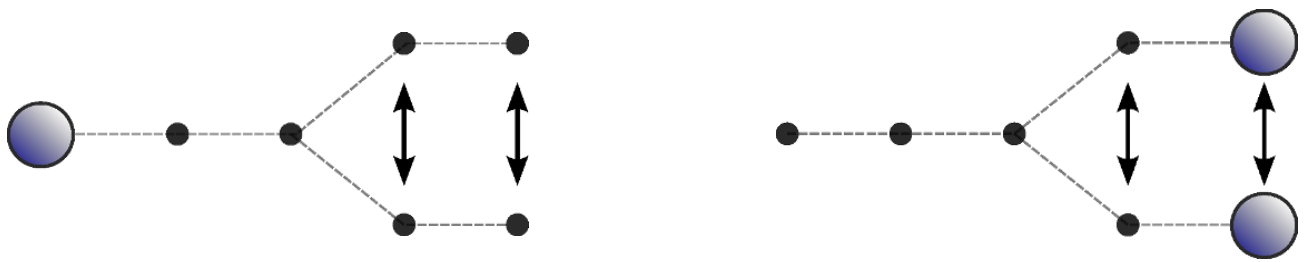

Figure 8. Different contractions of the $I V^{*}$ Kodaira fiber with $\mathbb{Z}_{2}$ monodromy (vertical arrows). Left: contract all components not intersecting the given section $\Rightarrow F_{4}$. Right: contract everything except the rightmost monodromy orbit $\Rightarrow B_{4}$.

matter content, one again expects the most simple degeneration of the Weierstrass model where the vanishing $\operatorname{degree}(\operatorname{deg} f, \operatorname{deg} g, \operatorname{deg} \Delta)$ jumps from $(3,4,8)$ to $(3,5,9)$. If one allows non-trivial monodromy, this requires a double intersection of the $I V^{*}$ and $I_{1}$ divisor components. Hence, by degree counting, there should be 14 such points and a numerical analysis of the Weierstrass hypersurface equation indeed confirms this. Unlike in the case without monodromy, there is no curve stuck over these codimension-two points in the base. Instead, the matter comes from one-parameter families of curves over the $I V^{*}$ discriminant locus and is determined by the Euler characteristic of their moduli space. In particular, the moduli space here is the base $\mathbb{P}^{1}$ branched at 14 points, that is, a Riemann surface of genus 6. To summarize, the generic Calabi-Yau hypersurface $Y_{2} \subset X_{\nabla_{2}}$ has Hodge numbers $h^{1,1}\left(Y_{2}\right)=6, h^{2,1}\left(Y_{2}\right)=180$. The 6 -d F-theory compactification is a $F_{4}$ gauge theory with $6 \times \underline{\mathbf{2 6}}$ matter hypermultiplets. ${ }^{20}$ This satisfies the gauge and gravitational anomaly cancellation conditions ${ }^{21}$

$$
\begin{gathered}
H_{u}=h^{2,1}+1-6 \cdot 2=169, \quad H_{c}=6 \cdot 26, \quad V=52 \\
H_{u}+H_{c}-V=273, \quad 18 b=6 \cdot 6-18, \quad 3 b^{2}=6 \cdot 3-15
\end{gathered}
$$

for $b=1$.

\subsection{Starting at the other end}

Instead of picking the fiber component intersecting the given section, we can also pick the two fiber components, exchanged by the monodromy, at the other end of the contracted Dynkin diagram (see figure 8). Shrinking all the complementary fiber components, we arrive at a $B_{4}$ gauge theory. We can use the extended-type $F_{4} \supset B_{4}$ branching rule

$$
\begin{aligned}
F_{4} & \supset B_{4}, \\
\operatorname{ad}\left(F_{4}\right) & =\operatorname{ad}\left(B_{4}\right) \oplus \underline{\mathbf{1 6}}, \\
\underline{\mathbf{2 6}} & =\underline{\mathbf{1 6}} \oplus \underline{\mathbf{9}} \oplus \underline{\mathbf{1}}
\end{aligned}
$$

to convert one into the other. To actually break the gauge group to $B_{4}$, the $\underline{\mathbf{1 6}}$ vector multiplet pairs up with a corresponding hypermultiplet and becomes massive. Hence we

\footnotetext{
${ }^{20}$ This matches the restricting of the anomaly virtual representation $H-V$ under the $E_{6} \supset F_{4}$ branching rule. Since the branching rule is of symmetric type, this breaking is not the traditional Higgs mechanism.

${ }^{21}$ Note that the charged dimension $[59,60]$ of $\underline{\mathbf{2 6}}$ is 24 . We review its definition in section A. In order to count a charged hyper as a complete $\underline{\mathbf{2 6}}$, we have to also remove two unchanged hypers.
} 
obtain a $B_{4}$ gauge theory with $5 \times \underline{\mathbf{1 6}}$ and $6 \times \underline{\mathbf{9}}$ charged hypermultiplets. The charged dimension of $\underline{\mathbf{9}}$ is only 8 , so we have to subtract 6 uncharged hypermultiplets to avoid overcounting. The resulting matter content cancels the gauge and gravity anomalies

$$
\begin{gathered}
H_{u}=h^{2,1}+1-6 \times 1=175, \quad H_{c}=5 \cdot 16+6 \cdot 9, \quad V=36 \\
H_{u}+H_{c}-V=273, \\
18 b=5 \cdot 4+6 \cdot 2-14, \quad 0=5 \cdot(-2)+6 \cdot 2-2, \quad 3 b^{2}=5 \cdot 3+6 \cdot 0-12
\end{gathered}
$$

for $b=1$.

More systematically, we can derive the hyper and vector multiplet count from the geometry of the elliptic fibration. Since identifying the correct branching rule will be somewhat tricky in an example that we encounter later on, let us walk through the more pedestrian argument. In the case with monodromy, note that there are no isolated codimension-two curves stuck over a point in the base and the proper way to count the non-isolated curves is by the genus of their moduli space. The trick is to identify all (possibly reducible) curves, count the number of massless fields supported on them, and then reassemble the component fields into gauge multiplets. For purposes of the exposition, let us stick to an $I V^{*}$ Kodaira fiber whose irreducible fiber components correspond to the simple $\widetilde{E}_{6}$ roots. The roots are generated by the simple roots, and we henceforth identify

- The 72 roots $\pm \alpha_{i}$ of $E_{6}$,

- The affine roots $\bigcup\{(\alpha ; 0),(\theta-\alpha ; 1)\}$ where $\theta$ is the highest root of $E_{6}$,

- Curves

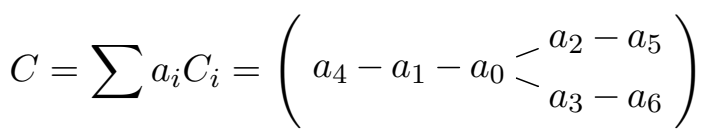

of self-intersection $C \cdot C=-2$ in $F$.

Clearly, the positive roots $\alpha_{i}$ correspond to the affine roots $\left(\alpha_{i} ; 0\right)$ which correspond to curves not wrapping the extended node in the Dynkin diagram. Flipping the sign of $\alpha_{i}$ corresponds to $\hat{\alpha}_{i} \mapsto(\theta, 1)-\hat{\alpha}_{i}$ which corresponds to $C \mapsto F-C$.

We are interested in the case where the gauge group is broken both by leaving some irreducible fiber components at finite size and by a monodromy group $\Gamma$ acting by permutation on the affine roots. A fiber component $C$ can be moved along the discriminant, and we take the moduli space to be a curve of genus $g^{\prime}\left(\mathcal{M}_{C}\right)$. We say that a curve shrinks if either $C$ or $F-C$ has zero volume, that is, does not wrap any irreducible fiber component chosen to have finite size.

- Each monodromy orbit $\Gamma C$ of a shrinking curve yields $g^{\prime}\left(\mathcal{M}_{C}\right)$ hypermultiplets and 1 vector multiplet.

- Each monodromy orbit $\Gamma C$ of a finite-size curve yields $g^{\prime}\left(\mathcal{M}_{C}\right)-1$ hypermultiplets and no vector multiplets. 


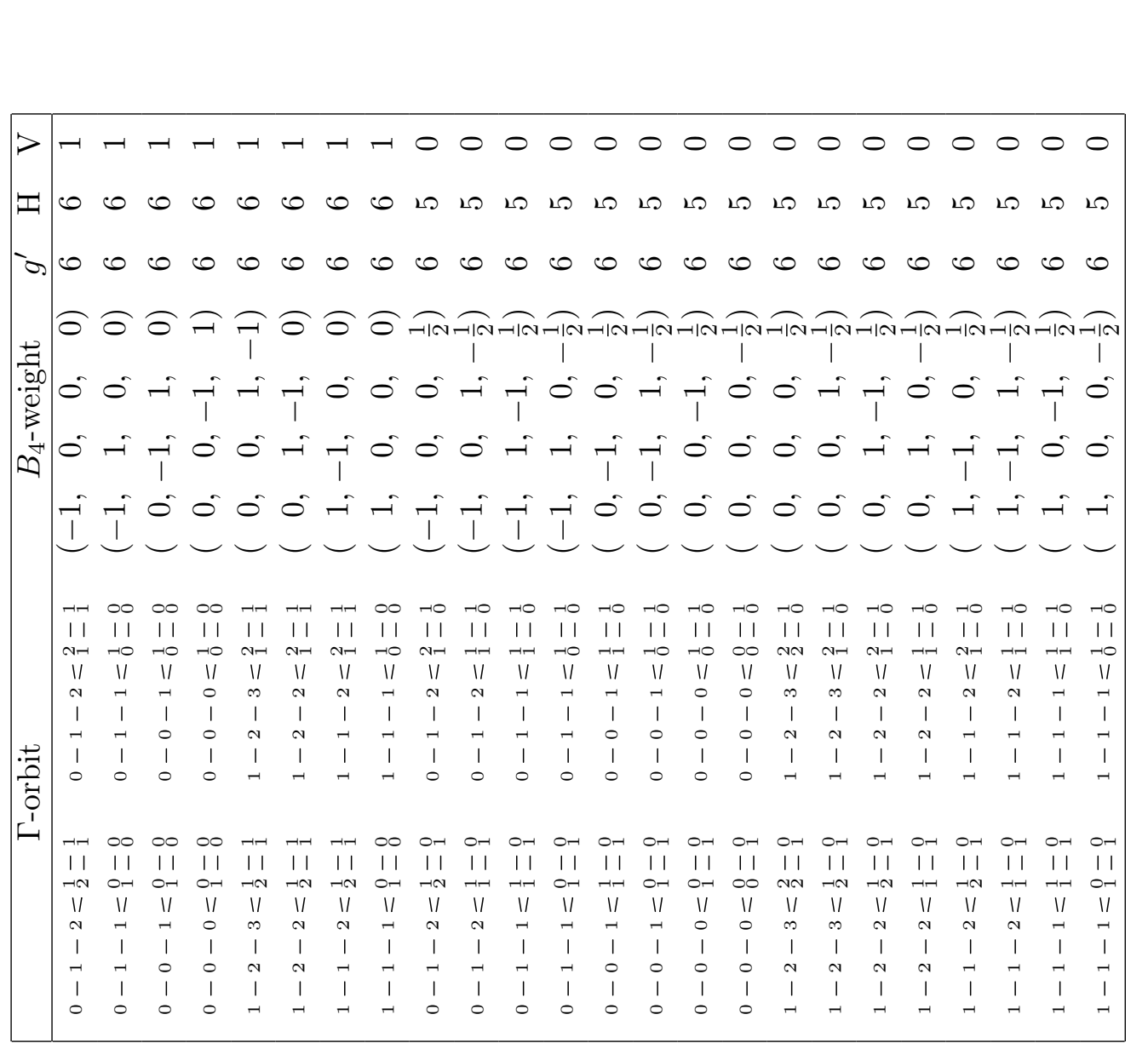

I 100000000000000000000000 - 000000000000000000000000

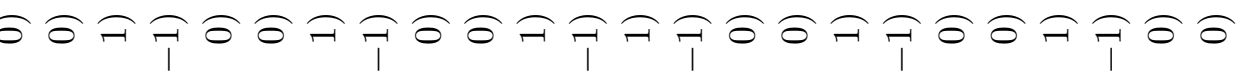
बुळ की

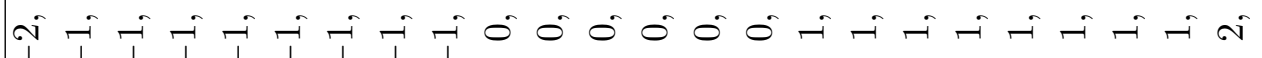
III I I I I I I

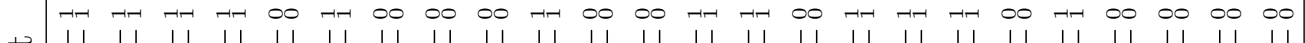

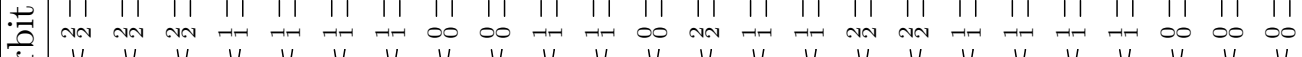

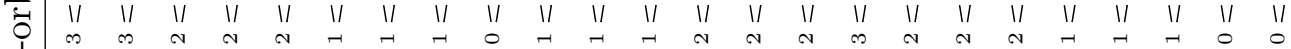

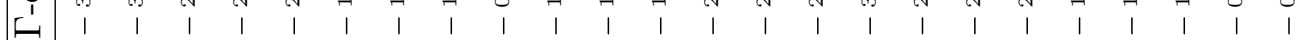

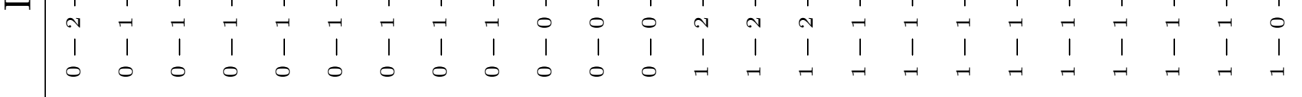


In the $B_{4}$ example, we distinguish two curve types: if the curve is fixed by the monodromy, then its moduli space equals the corresponding discriminant component (which is a $\mathbb{P}^{1}$ ). If the fiber component is exchanged by monodromy, then it is a branched double cover of $\mathbb{P}^{1}$. The number of branch points can be read off from the Weierstrass model, and we find 14. Hence, the moduli space of curves that are not fixed under the monodromy action is a Riemann surface of genus $g^{\prime}=6$. The roots are tabulated in table 3 . We see that, indeed, the spectrum consists of a vector multiplet in the adjoint of $B_{4}$ as well as $5 \times \underline{\mathbf{1 6}}$ and $6 \times \underline{\mathbf{9}}$ hypermultiplets. Finally, we can also systematically count the number of uncharged hypermultiplets. The weight zero subspace of the adjoint of $E_{8}$ is 6 , which means that this many complex structure moduli are actually frozen by requiring a $I V^{*}$ Kodaira fiber. This is already accounted for in the gravitational anomaly where the complete $V$ including its weight zero subspace is subtracted from the number of complex structure moduli. To assemble the components in table 3 into complete $B_{4}$ representations, we need 4 uncharged fields for the adjoint and one uncharged field for the $\underline{\mathbf{9}}$. The adjoint field is the gauge vector multiplet, and its weight zero subspace is already accounted for. Therefore, the remaining number of $B_{4}$-uncharged hypermultiplets equals

$$
H_{u}=h^{2,1}+1-6 \cdot 1=175 \quad \Rightarrow \quad H_{u}+H_{c}-V=273 .
$$

\subsection{A novel monodromy effect}

The third model with a $I V^{*}$ Kodaira fiber under consideration will have a different fiber ambient space, namely $\mathbb{P}^{2}$ instead of $\mathbb{P}^{2}[1,2,3]$. The effect of this change is that the toric ambient space no longer forces the fibration to have a section but only 3-sections. Explicitly, we consider the polytope $\nabla_{3}$ with points

\begin{tabular}{|c|c|ccc|ccc|c|}
\hline$u$ & $v$ & $w_{0}$ & $w_{1}$ & $w_{2}$ & $f_{0}$ & $f_{1}$ & $f_{2}$ & \\
\hline 0 & -1 & 1 & 1 & 1 & 1 & 0 & -1 & 0 \\
0 & 1 & 0 & 0 & 0 & 0 & 1 & -1 & 0 \\
-1 & 0 & 3 & 2 & 1 & 0 & 0 & 0 & 1 \\
-1 & 1 & 0 & 0 & 0 & 0 & 0 & 0 & 0 \\
\hline \multicolumn{7}{|c|}{ three tops } & \multicolumn{3}{c}{ fiber } & facet interior \\
\hline
\end{tabular}

where again the only non-trivial discriminant component comes from the $w$-top. Each of $V\left(f_{0}\right), V\left(f_{1}\right)$, and $V\left(f_{2}\right)$ is a toric 3 -section. From the intersection numbers we conclude that, restricted to a generic fiber $F, V\left(w_{0}\right) \cap F$ consists of a single $\mathbb{P}^{1}$ of multiplicity 3 , $V\left(w_{1}\right) \cap F$ consists of three disjoint $\mathbb{P}^{1}$ of multiplicity 2 , and $V\left(w_{2}\right) \cap F$ consists of three disjoint $\mathbb{P}^{1}$ of multiplicity 1 . Therefore, the affine $E_{6}$ Dynkin diagram is folded as in figure 9 into the ambient toric divisors. Each of the three disconnected components of $V\left(w_{2}\right) \cap F$ intersects the irreducible three-section $V\left(f_{0}\right)$ in a point. Hence, the monodromy action freely permutes the three irreducible components in $V\left(w_{2}\right) \cap F$. In particular, there cannot be a section (toric or not) in this genus-one fibration as it would unambiguously mark one of the three components.

As discussed in section 5 , the lack of a section allows for nodes in the $I_{1}$ discriminant component. In fact, a numerical analysis of a generic Calabi-Yau hypersurface finds that 

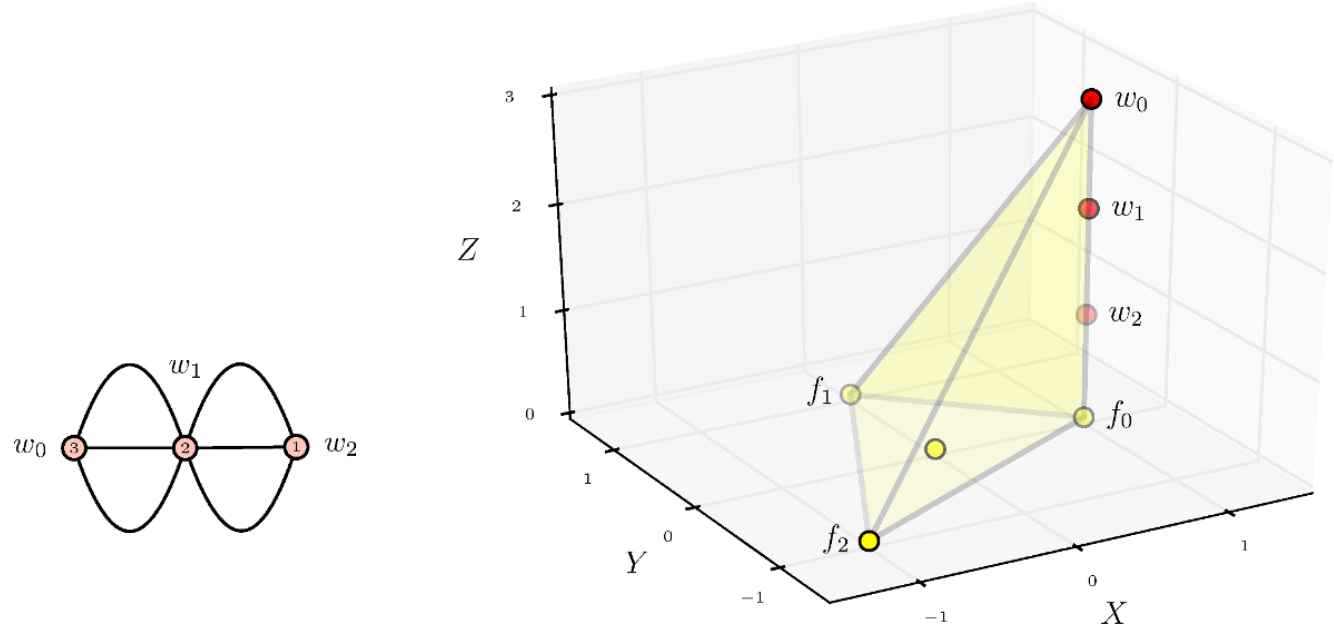

Figure 9. The $G_{2}$ top with $\mathfrak{S}_{3}$-monodromy and associated Dynkin diagram.

there are 108 nodes in the $I_{1}$ discriminant component, all of which are away from the $I V^{*}$ discriminant component. This contributes an extra 108 uncharged hypermultiplets localized in codimension-two over the base.

For any gauge theory to arise, one needs to contract some of the fiber irreducible components. Since there is no section, the standard prescription cannot be applied. The minimal generalization would be to contract all three irreducible components $V\left(w_{2}\right) \cap F$, and this is what we will consider in the remainder of this subsection. This corresponds to the $E_{6} \supset G_{2}$ branching rule, the composition of $E_{6} \supset D_{4}$ from eq. (7.4) with the symmetric-type $D_{4} \supset G_{2}$ branching rule. There is no change in the Weierstrass model compared to the nonsplit $E_{6}$, so we still expect 14 branch points over the $I V^{*}$ discriminant locus. This is confirmed by a numerical analysis of a generic Calabi-Yau hypersurface. There are three possible stabilizers under the symmetric group action, leading to the following types of roots:

- The moduli space of a $\mathfrak{S}_{3}$-symmetric curve is still $\mathbb{P}^{1}$. For example, the moduli space of the central fiber component $C_{0}=V\left(w_{0}\right) \cap F$ is still $\mathbb{P}^{1}$.

$$
0-0-1=0-0
$$

- The moduli space of a $\mathbb{Z}_{2}$-invariant curve is a triple cover of $\mathbb{P}^{1}$ branched at 14 points. Such a Riemann surface has genus $g^{\prime}=5$. For example, $C_{1}$ which is one of the three $0-1-0 \leq \begin{aligned} & 0-0 \\ & 0-0\end{aligned}$ irreducible components of $V\left(w_{1}\right) \cap F$.

- The moduli space of a curve with trivial $\mathfrak{S}_{3}$-stabilizier is a sixfold cover over $\mathbb{P}^{1}$ branched at 14 points. At each ramification point, the six sheets meet in three pairs. Hence, $1-1-2 \leq \begin{aligned} & 1-0 \\ & 2-1\end{aligned}$ the moduli space is a Riemann surface of genus $g^{\prime}=16$.

The spectrum is tabulated in table 4 using the method explained in section 7.4. Reassem-

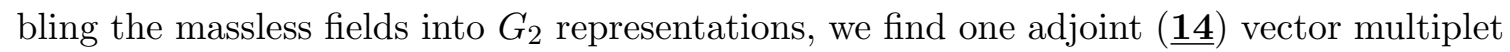




\begin{tabular}{|c|c|c|c|c|c|c|}
\hline \multicolumn{3}{|c|}{$\Gamma$-orbit } & \multicolumn{2}{|c|}{$G_{2 \text {-wt. }} \quad g^{\prime}(C)$} & \multirow{2}{*}{$\begin{array}{c}\mathrm{H} \\
0\end{array}$} & \multirow{2}{*}{$\begin{array}{l}\mathrm{V} \\
1\end{array}$} \\
\hline & $1-1-2=\frac{1}{1}=\frac{1}{1}$ & & $(-3,3)$ & 0 & & \\
\hline & $0-0-1=\begin{array}{l}0 \\
0\end{array}=\begin{array}{l}0 \\
0\end{array}$ & & $(-3,6)$ & 0 & 0 & 1 \\
\hline & $1-1-1=\frac{1}{1}-\frac{1}{1}$ & & $(0,-3)$ & 0 & 0 & 1 \\
\hline & $0-1-2=\frac{1}{1}=0$ & & $(0,3)$ & 0 & 0 & 1 \\
\hline & $1-2-2=\frac{2}{2}-\frac{1}{1}$ & & $(3,-6)$ & 0 & 0 & 1 \\
\hline & $0-1-1=\frac{1}{1}=0$ & & $(3,-3)$ & 0 & 0 & 1 \\
\hline $1-1-3=\frac{2}{2}=\frac{1}{1}$ & $1-2-3=\frac{1}{2}-\frac{1}{1}$ & $1-2-3=\frac{2}{1}=\frac{1}{1}$ & $(-2,3)$ & 5 & 5 & 1 \\
\hline $1-1-2=\frac{1}{2}=\frac{1}{1}$ & $1-1-2={ }_{1}^{2}=\frac{1}{1}$ & $1-2-2=\frac{1}{1}-\frac{1}{1}$ & $\left(\begin{array}{ll}-1, & 0\end{array}\right)$ & 5 & 5 & 1 \\
\hline $0-0-1=\begin{array}{l}0 \\
1\end{array}-0$ & $0-0-1=\begin{array}{l}1 \\
0\end{array}-0$ & $0-1-1=\begin{array}{l}0 \\
0\end{array}-0$ & $(-1,3)$ & 5 & 5 & 1 \\
\hline $1-1-2=\frac{2}{2}-\frac{1}{1}$ & $1-2-2=\frac{1}{2}-\frac{1}{1}$ & $1-2-2={ }_{1}^{2}-\frac{1}{1}$ & $(1,-3)$ & 5 & 5 & 1 \\
\hline $0-0-1=\frac{1}{1}=0$ & $0-1-1=0 \begin{array}{l}0 \\
1\end{array}-0$ & $0-1-1={ }_{0}^{1}=\stackrel{0}{0}$ & $\left(\begin{array}{ll}1, & 0\end{array}\right)$ & 5 & 5 & 1 \\
\hline $0-0-0={ }_{1}^{0}=0$ & $0-0-0={ }_{0}^{1}=0$ & $0-1-0=\begin{array}{l}0 \\
0\end{array}=\begin{array}{l}0 \\
0\end{array}$ & $(2,-3)$ & 5 & 5 & 1 \\
\hline $0-0-1={ }_{1}^{0}-\frac{0}{1}$ & $0-0-1=\frac{1}{0}=\frac{1}{0}$ & $1-1-1={ }_{0}^{0}=0$ & $(-2,3)$ & 5 & 4 & 0 \\
\hline $0-1-2=\frac{1}{1}=\frac{1}{1}$ & $1-1-2=\frac{1}{1}=\frac{0}{1}$ & $1-1-2=\frac{1}{1}=\frac{1}{0}$ & $(-2,3)$ & 5 & 4 & 0 \\
\hline $0-0-0=0 \begin{array}{l}0 \\
0\end{array}=\stackrel{0}{1}$ & $0-0-0={ }_{0}^{0}=\frac{1}{0}$ & $1-0-0=\begin{array}{l}0 \\
0\end{array}=0$ & $(-1, \quad 0)$ & 5 & 4 & 0 \\
\hline $0-0-1=\frac{1}{1}=\frac{1}{1}$ & $1-1-1=0 \begin{array}{l}0 \\
1\end{array}=0$ & $1-1-1=\frac{1}{0}=\frac{1}{0}$ & $(-1, \quad 0)$ & 5 & 4 & 0 \\
\hline $0-1-2=\frac{1}{1}=\frac{0}{1}$ & $0-1-2=\frac{1}{1}-\frac{1}{0}$ & $1-1-2=\frac{1}{1}=0$ & $(-1,3)$ & 5 & 4 & 0 \\
\hline $0-1-3=\frac{2}{2}=\frac{1}{1}$ & $1-2-3=\frac{1}{2}=\stackrel{0}{1}$ & $1-2-3={ }_{1}^{2}=\frac{1}{0}$ & $(-1,3)$ & 5 & 4 & 0 \\
\hline $0-0-0=\begin{array}{l}0 \\
1\end{array}=0$ & $0-0-0={ }_{0}^{1}=\frac{1}{0}$ & $1-1-0=\begin{array}{l}0 \\
0\end{array}=0$ & $(1,-3)$ & 5 & 4 & 0 \\
\hline $0-1-1=\frac{1}{1}=\frac{1}{1}$ & $1-1-1=\frac{1}{1}=0$ & $1-1-1=\frac{1}{1}=\frac{1}{0}$ & $(1,-3)$ & 5 & 4 & 0 \\
\hline $0-1-2=\frac{1}{2}=0$ & $0-1-2={ }_{1}^{2}=\frac{1}{0}$ & $1-2-2=\frac{1}{1}=0$ & $(1,0)$ & 5 & 4 & 0 \\
\hline $0-2-3=\frac{2}{2}=\frac{1}{1}$ & $1-2-3=\frac{2}{2}=0$ & $1-2-3=\frac{2}{2}=\frac{1}{0}$ & $(1,0)$ & 5 & 4 & 0 \\
\hline $0-1-1=\frac{1}{1}=\stackrel{0}{1}$ & $0-1-1=\frac{1}{1}=\frac{1}{0}$ & $1-1-1=\frac{1}{1}=0$ & $(2,-3)$ & 5 & 4 & 0 \\
\hline $0-1-2=\frac{2}{2}=\frac{1}{1}$ & $1-2-2=\frac{1}{2}=0$ & $1-2-2=2=1$ & $(2,-3)$ & 5 & 4 & 0 \\
\hline $\begin{array}{l}0-0-1=1=0 \\
0-1-1=1=1 \\
=0\end{array}$ & $\begin{array}{l}0-1-1=0=0 \\
1-1-1=00=0 \\
1=0\end{array}$ & $\begin{array}{l}0-0-1=1=1 \\
1-1-1=1=0 \\
0=0\end{array}$ & $\left(\begin{array}{ll}0, & 0\end{array}\right)$ & 16 & 15 & 0 \\
\hline $\begin{array}{l}0-1-2=\frac{1}{2}=1 \\
1-2-2=1-0 \\
1-1\end{array}$ & $\begin{array}{l}0-1-2=2-1 \\
1-1-2=1-1 \\
1-0\end{array}$ & $\begin{array}{l}1-1-2=\frac{1}{2}-0 \\
1-2-2=1-1 \\
1-0\end{array}$ & $\left(\begin{array}{ll}0, & 0\end{array}\right)$ & 16 & 15 & 0 \\
\hline
\end{tabular}

Table 4. The $\Gamma=\mathfrak{S}_{3}$-orbits of the 72 curves, $G_{2}$ weight, genus of the moduli space, and the resulting number of hyper and vector multiplets. The weight under $G_{2}$ is the intersection product with $C_{1}+C_{2}+C_{3}$ and $3 C_{0}$. 
and $5+4+4=13$ hypermultiplets transforming in the the fundamental $\underline{\mathbf{7}}$. There are also $15+15=30$ additional uncharged hypermultiplets. To summarize, the generic Calabi-Yau hypersurface $Y_{3} \subset X_{\nabla_{3}}$ has Hodge numbers $h^{1,1}\left(Y_{3}\right)=4, h^{2,1}\left(Y_{3}\right)=70$. The 6-d F-theory compactification is a $G_{2}$ gauge theory with $13 \times \underline{\mathbf{7}}$ matter hypermultiplets. This satisfies the gauge and gravity anomaly cancellation conditions ${ }^{22}$

$$
\begin{gathered}
H_{u}=h^{2,1}+1-13 \cdot 1+30+108=196, \quad H_{c}=13 \cdot 7, \quad V=14 \\
H_{u}+H_{c}-V=273, \quad 18 b=13 \cdot 2-8, \quad 3 b^{2}=13 \cdot 1-10
\end{gathered}
$$

for $b=1$.

\subsection{Alternative limits}

There is no particular reason to pick the monodromy orbit at the ends of the affine $E_{6}$ Dynkin diagram as the curves to keep at finite size. Certainly, there is no given section that would single out this choice. Another possibility is to select the central (monodromyinvariant) node. As we will see, this choice leads to a 5 -d theory with some interesting features, and suggests similar features of the 6-d F-theory limit.

First, the unbroken gauge group is $A_{2}=\mathrm{SU}(3)$ : not shrinking the central node yields an $A_{2}^{3}$ gauge group as discussed in section 7.2. The $\mathfrak{S}_{3}$ monodromy breaks it further to the diagonal $A_{2}$. Note that the monodromy is the same as in section 7.5 , only the choice of shrinking curves differs. Breaking the anomaly virtual representation by this branching rule leads to

$$
H-V=7 \times \underline{\mathbf{2 7}} \ominus \operatorname{ad}\left(E_{6}\right)=14 \times \operatorname{ad}(\operatorname{SU}(3)) \oplus 19 \times \underline{\mathbf{1}} \ominus \operatorname{Sym}^{3}(\underline{\mathbf{3}}) \ominus \operatorname{Sym}^{3}(\underline{\overline{\mathbf{3}}}) .
$$

Surprisingly, this representation content is at odds with Witten's rule [61] for counting fields by the genus of the moduli space of the curves (as reviewed in section 7.4). In particular, the naïve application would result in the same SU(3)-representations for vectors and hypers in contrast to the branching rule. The resolution of this puzzle must be that there are additional massless degrees of freedom, and indeed there ought to be: the central $\mathbb{P}^{1}$ fiber component, which we chose to leave at finite size, has multiplicity three. Therefore, one has additional infinitesimal ${ }^{23}$ deformations that ought to manifest themselves as massless fields.

We can understand this multiplicity in more detail by writing down the projection map. For the smooth Calabi-Yau hypersurface, this is

$$
X_{\Delta_{3}} \rightarrow \mathbb{P}^{2}, \quad\left[u: v: w_{0}: w_{1}: w_{2}: f_{1}: f_{2}: f_{3}\right] \mapsto\left[u: v: w_{0}^{3} w_{1}^{2} w_{2}\right]
$$

for any smooth triangulation of the polytope eq. (7.13) respecting the fibration structure. Now, contracting all fiber components except the central node amounts to removing the rays corresponding to $w_{1}$ and $w_{2}$ from the fan and merging their star into a single cone, making the triangulation coarser. The projection map of this singular toric variety is

$$
X_{\Delta_{3}^{\prime}} \rightarrow \mathbb{P}^{2}, \quad\left[u: v: w_{0}: f_{1}: f_{2}: f_{3}\right] \mapsto\left[u: v: w_{0}^{3}\right]
$$

\footnotetext{
${ }^{22}$ Similar to eq. (7.8), note that the charged dimension of $\underline{\mathbf{7}}$ is 6 .

${ }^{23}$ They do not extend to finite deformations, which means that there is a superpotential term that prevents them from acquiring a vev.
} 


\begin{tabular}{|c|c|c|c|c|c|c|}
\hline & \multirow{2}{*}{$\begin{array}{c}\Gamma \text {-orbit } \\
1-1-2=\frac{1}{1}=\frac{1}{1}\end{array}$} & & $A_{2}$-wt. & \multirow{2}{*}{$\frac{g^{\prime}}{0}$} & \multirow{2}{*}{$\begin{array}{c}\mathrm{H} \\
0\end{array}$} & \multirow{2}{*}{$\begin{array}{l}\mathrm{V} \\
1\end{array}$} \\
\hline & & & $(-3, \quad 3)$ & & & \\
\hline & $0-0-1=\begin{array}{l}0 \\
0\end{array}=0$ & & $(-3, \quad 0)$ & 0 & 0 & 1 \\
\hline & $1-1-1=\frac{1}{1}=\frac{1}{1}$ & & $(0,3)$ & 0 & 0 & 1 \\
\hline & $0-1-2=\frac{1}{1}=0$ & & $(0,-3)$ & 0 & 0 & 1 \\
\hline & $1-2-2=\frac{2}{2}=\frac{1}{1}$ & & $\left(\begin{array}{ll}3, & 0\end{array}\right)$ & 0 & 0 & 1 \\
\hline & $0-1-1=\frac{1}{1}=0$ & & $(3,-3)$ & 0 & 0 & 1 \\
\hline $1-1-3=\frac{2}{2}=\frac{1}{1}$ & $1-2-3=\frac{1}{2}=\frac{1}{1}$ & $1-2-3={ }_{1}^{2}=\frac{1}{1}$ & $(-2, \quad 1)$ & 5 & 6 & 2 \\
\hline $1-1-2=\frac{1}{2}=\frac{1}{1}$ & $1-1-2=2 \frac{1}{1}=\frac{1}{1}$ & $1-2-2=\frac{1}{1}=\frac{1}{1}$ & $(-1, \quad 2)$ & 5 & 5 & 1 \\
\hline $0-0-1=\begin{array}{l}0 \\
1\end{array}=0$ & $0-0-1={ }_{0}^{1}=0$ & $0-1-1=\begin{array}{l}0 \\
0\end{array}=0$ & $(-1,-1)$ & 5 & 5 & 1 \\
\hline $1-1-2=\frac{2}{2}=\frac{1}{1}$ & $1-2-2=\frac{1}{2}=\frac{1}{1}$ & $1-2-2=\frac{2}{1}=1$ & $(1,1)$ & 5 & 5 & 1 \\
\hline $0-0-1=\frac{1}{1}=0$ & $0-1-1=0 \begin{array}{l}0 \\
1\end{array}-0$ & $0-1-1=\frac{1}{0}=0$ & $(1,-2)$ & 5 & 5 & 1 \\
\hline $0-0-0=\begin{array}{l}0 \\
1\end{array}=0 \begin{array}{c}0 \\
0\end{array}$ & $0-0-0=\frac{1}{0}=0$ & $0-1-0=\begin{array}{l}0 \\
0\end{array}=\begin{array}{l}0 \\
0\end{array}$ & $(2,-1)$ & 5 & 6 & 2 \\
\hline $0-0-1=0 \begin{array}{l}0 \\
1\end{array}$ & $0-0-1={ }_{0}^{1}=\frac{1}{0}$ & $1-1-1={ }_{0}^{0}=0$ & $(-2, \quad 1)$ & 5 & 4 & 0 \\
\hline $0-1-2=\frac{1}{1}=\frac{1}{1}$ & $1-1-2=\frac{1}{1}=\begin{array}{l}0 \\
1\end{array}$ & $1-1-2=\frac{1}{1}=0$ & $(-2, \quad 1)$ & 5 & 4 & 0 \\
\hline $0-0-0=0 \begin{array}{l}0 \\
0\end{array}$ & $0-0-0={ }_{0}^{0}={ }_{0}^{1}$ & $1-0-0=\begin{array}{l}0 \\
0\end{array}=\begin{array}{l}0 \\
0\end{array}$ & $(-1, \quad 2)$ & 5 & 5 & 1 \\
\hline $0-0-1=\frac{1}{1}=\frac{1}{1}$ & $1-1-1=\begin{array}{l}0 \\
1\end{array}=\begin{array}{l}0 \\
1\end{array}$ & $1-1-1={ }_{0}^{1}=\frac{1}{0}$ & $(-1, \quad 2)$ & 5 & 4 & 0 \\
\hline $0-1-2=\frac{1}{1}=\begin{array}{l}0 \\
1\end{array}$ & $0-1-2=\frac{1}{1}=\frac{1}{0}$ & $1-1-2=\frac{1}{1}=0$ & $(-1,-1)$ & 5 & 4 & 0 \\
\hline $0-1-3=\frac{2}{2}=\frac{1}{1}$ & $1-2-3=\frac{1}{2}=\stackrel{0}{1}$ & $1-2-3={ }_{1}^{2}=\frac{1}{0}$ & $(-1,-1)$ & 5 & 5 & 1 \\
\hline $0-0-0=0 \begin{array}{l}0 \\
1\end{array}=0$ & $0-0-0={ }_{0}^{1}={ }_{0}^{1}$ & $1-1-0=\begin{array}{l}0 \\
0\end{array}=0$ & $(1,1)$ & 5 & 5 & 1 \\
\hline $0-1-1=\frac{1}{1}=\frac{1}{1}$ & $1-1-1=\frac{1}{1}=\begin{array}{l}0 \\
1\end{array}$ & $1-1-1=\frac{1}{1}=\frac{1}{0}$ & $(1,1)$ & 5 & 4 & 0 \\
\hline $0-1-2=\frac{1}{2}=\frac{0}{1}$ & $0-1-2=2=1$ & $1-2-2=\frac{1}{1}=0$ & $(1,-2)$ & 5 & 4 & 0 \\
\hline $0-2-3=\frac{2}{2}=\frac{1}{1}$ & $1-2-3=\frac{2}{2}=\frac{0}{1}$ & $1-2-3=\frac{2}{2}=\frac{1}{0}$ & $(1,-2)$ & 5 & 5 & 1 \\
\hline $0-1-1=\frac{1}{1}=\frac{0}{1}$ & $0-1-1=\frac{1}{1}=\frac{1}{0}$ & $1-1-1=\frac{1}{1}=0$ & $(2,-1)$ & 5 & 4 & 0 \\
\hline $0-1-2=\frac{2}{2}=\frac{1}{1}$ & $1-2-2=\frac{1}{2}=0$ & $1-2-2={ }_{1}^{2}=\frac{1}{0}$ & $(2,-1)$ & 5 & 4 & 0 \\
\hline $\begin{array}{l}0-0-1=1=0 \\
0-1-1=1=1 \\
0=0\end{array}$ & $\begin{array}{l}0-1-1=0=0 \\
1-1-1=0=0 \\
1=0\end{array}$ & $\begin{array}{l}0-0-1=\frac{1}{1}=1 \\
1-1-1=1 \\
0-0\end{array}$ & $\left(\begin{array}{ll}0, & 0\end{array}\right)$ & 16 & 15 & 0 \\
\hline $\begin{array}{l}0-1-2=\frac{1}{2}=1 \\
1-2-2=\frac{1}{1}=1 \\
1\end{array}$ & $\begin{array}{l}0-1-2=1=1 \\
1-1-2=1=0\end{array}$ & $\begin{array}{l}1-1-2=\frac{1}{2}=0 \\
1-2-2=1=1 \\
1-0\end{array}$ & $\left(\begin{array}{ll}0, & 0\end{array}\right)$ & 16 & 15 & 0 \\
\hline
\end{tabular}

Table 5. The $\Gamma=\mathfrak{S}_{3}$-orbits of the 72 curves, $A_{2}$ weight, genus of the moduli space, and the resulting number of hyper and vector multiplets. The weight under $A_{2}$ is the intersection product with $C_{1}+C_{2}+C_{3}$ and $C_{4}+C_{5}+C_{6}$.

and we see that turning on $w_{0}$ infinitesimally does indeed not move the fiber to first order. We note that this is a novel feature of not having a section: a section can only intersect the fiber in a single point (counted with multiplicity), that is, a point on a fiber component with multiplicity one. Only a three-section can intersect the central node of the affine $E_{6}$ in a single point of multiplicity three. Hence, we propose the following addition to the Witten's rule for counting the massless spectrum:

- Each curve $C$ that does not wrap a fiber irreducible component whose multiplicity is one yields an additional hyper/vector multiplet pair. 
Note that, as before, this is meant to apply to curves such that either $C$ or $F-C$ does not wrap a multiplicity-one curve, that is, either $a_{4}=a_{5}=a_{6}=0$ or $a_{4}=a_{5}=a_{6}=1$. In table 5 we applied this modified rule to count the hyper- and vector multiplets.

In terms of $A_{2}$-representations, the weights naïvely assemble into $\operatorname{Sym}^{3}(\underline{\mathbf{3}}) \oplus \operatorname{Sym}^{3}(\underline{\overline{\mathbf{3}}})$ of vector multiplets and vector and 14 adjoint hypermultiplets, which is at first sight quite an odd field content. However, also note that the weights live in an index-3 sublattice of the $A_{2}$ weight lattice. Geometrically, this is because $\left(C_{1}+C_{2}+C_{3}\right)-\left(C_{4}+C_{5}+C_{6}\right)$ is divisible by 3 modulo $F=3 C_{0}+2\left(C_{1}+C_{2}+C_{3}\right)+\left(C_{4}+C_{5}+C_{6}\right)$. Hence, we should have used these refined curve classes which amounts to the intersection numbers spanning the whole weight lattice instead of a index-3 sublattice. Hence, we identify the actual field content as vector multiplets transforming in the ad $(\mathrm{SU}(3)) \oplus 2 \times(\underline{\mathbf{3}} \oplus \underline{\overline{\mathbf{3}}})$ and hypermultiplets transforming as $14 \times(\underline{\mathbf{3}} \oplus \underline{\overline{\mathbf{3}}})$. The vector multiplets not in the adjoint can pair up with hypermultiplets and become massive, leaving us with an ordinary $\mathrm{SU}(3)$ gauge theory with $12 \times(\underline{\mathbf{3}} \oplus \underline{\overline{\mathbf{3}}})$ charged hypermultiplets, canceling the non-Abelian gauge anomaly

$$
18 b=12 \cdot(1+1)-6, \quad 3 b^{2}=12 \cdot\left(\frac{1}{2}+\frac{1}{2}\right)-9
$$

for $b=1$. To count the uncharged hypermultiplets, note that there are again $2 \times 15$ uncharged fields localized along the discriminant, see table 5, and 108 fields localized in codimension-two at the cusps of the $I_{1}$ discriminant component. Together, they cancel the gravitational anomaly as

$$
H_{u}=h^{2,1}+1+30+108, \quad H_{c}=12 \cdot(3+3), \quad V=8, \quad H_{u}+H_{c}-V=273 .
$$

\section{The Shioda-Tate(-Wazir) formula}

The divisors in a smooth elliptic fibration $\pi: Y \rightarrow B$ are either (multi-)sections, that is, map to all of $B$, or vertical, that is, their image is again codimension-one in the base. The vertical case can be further subdivided in the case where divisor contains the entire fiber or only irreducible fiber components. In particular, one has the following divisors:

- The given section $\sigma$,

- sections $S_{i}, i=1, \ldots, \operatorname{rank} M W(Y)$ forming a basis of the Mordell-Weil group,

- Pull-backs from the base, $B_{j}=\pi^{-1}\left(b_{j}\right), j=1, \ldots, h^{1,1}(Y)$,

- and fibral divisors $T_{\delta, k}$ that are irreducible components of the preimage $\pi^{-1}(\delta)$ of irreducible components of the discriminant, $\delta \subset \Delta$. The subscript $k=0, \ldots, K_{\delta}-1$ labels the irreducible fiber components modulo the monodromy action.

To avoid obvious homology relations, we considered a basis for the Mordell-Weil group and the base divisors $b_{j}$ here. For the fibral divisors $T_{\delta, k}$ one further notes that the sum over all fiber components is already generated by the $B_{j}$, so we have to exclude one of the fiber components. Customarily, one excludes the component intersecting the given section 
(labeled $k=0$ ), so we demand that $k \geq 1$. The Shioda-Tate formula [68-70] (extended to threefolds by Wazir [71]) statess that these generate all divisor classes, that is,

$$
h^{1,1}(Y)=1+\operatorname{rank} M W(Y)+h^{1,1}(B)+\sum_{\delta}\left(K_{\delta}-1\right)
$$

Now consider a genus-one fibration $Y^{\prime} \rightarrow B$ without a section. Even if there is no section, there is always some multi-section $\sigma^{\prime}$ for a sufficiently high degree for a projective genus-one fibration, generating some one-dimensional subspace of $h^{1,1}\left(Y^{\prime}\right)$. Hence, the generalization of Shioda-Tate-Wazir formula still contains the " $1+$ " part.

As for the fibral divisors, the story also generalizes in an obvious way. Even if there are more general monodromies, one still has to group irreducible fiber components into the $K_{\delta}^{\prime}$ monodromy orbits. One of these fibral divisors needs to be excluded to avoid homology relations with the divisors $B_{j}$. We cannot rely on a section to pick it, but any choice is fine.

Finally, consider the term involving the Mordell-Weil group. For a genus-one fibration $Y^{\prime}$ we still have an associated Mordell-Weil group $M W\left(J\left(Y^{\prime}\right)\right)$ of the Jacobian. Recall that the Mordell-Weil group acts by translations (birationally) on the points of the elliptic fibration. This action commutes with the twist by an element of the Tate-Shafarevich group, and therefore extends to an action on the genus-one fibration. Hence we can act on the chosen multi-section $\sigma^{\prime}$ to produce new multi-sections $S_{i}^{\prime}\left(\sigma^{\prime}\right)$ of the same degree. Moreover, free generators in the Mordell-Weil group cannot fix $\sigma^{\prime}$. In fact, the set of images $S_{i}^{\prime}\left(\sigma^{\prime}\right)$ for a basis $i=1, \ldots, \operatorname{rank} M W\left(J\left(Y^{\prime}\right)\right)$ are again independent multi-sections in homology.

To summarize, the Shioda-Tate-Wazir formula generalized to

$$
h^{1,1}\left(Y^{\prime}\right)=1+\operatorname{rank} M W\left(J\left(Y^{\prime}\right)\right)+h^{1,1}(B)+\sum_{\delta}\left(K_{\delta}^{\prime}-1\right)
$$

for genus-one fibrations. By the analogous argument as in the case with a section, we can identify the Mordell-Weil lattice with the Abelian gauge bosons in the F-theory compactification. That is, the F-theory gauge group is $G \times \mathrm{U}(1)^{r}$ with $r=\operatorname{rank} M W(J(Y))$ and $G$ a simple Lie group.

\section{Acknowledgments}

We thank Lara Anderson, Paul Aspinwall, Andreas Braun, Andrés Collinucci, Daniel Bump, Antonella Grassi, Simeon Hellerman, Kentaro Hori, Gregory Moore, Nathan Seiberg, Washington Taylor, and Edward Witten for discussions. We gratefully acknowledge the hospitality of the Simons Center for Geometry and Physics as well as the University of Pennsylvania where part of this work was performed; D.R.M. also thanks the Aspen Center for Physics for hospitality. The work of D.R.M. was supported in part by the National Science Foundation under grants DMS-1007414, PHY-1066293, and PHY-1307513. The work of V.B. was supported in part by the Dublin Institute for Advanced Studies and by the EPSRC grant BKRWDM00. 


\section{A Representation theory in sage}

The representation theory of semisimple Lie groups is of course a well-known subject. However the richness of representations and branchings often requires us to look through tables, which is quite tedious. In this appendix we would like to give a quick introduction to Sage $[72,73]$ (http://www.sagemath.org) and show how one can use it to compute with representations. Let us start by defining the adjoint representation of $E_{6}$ :

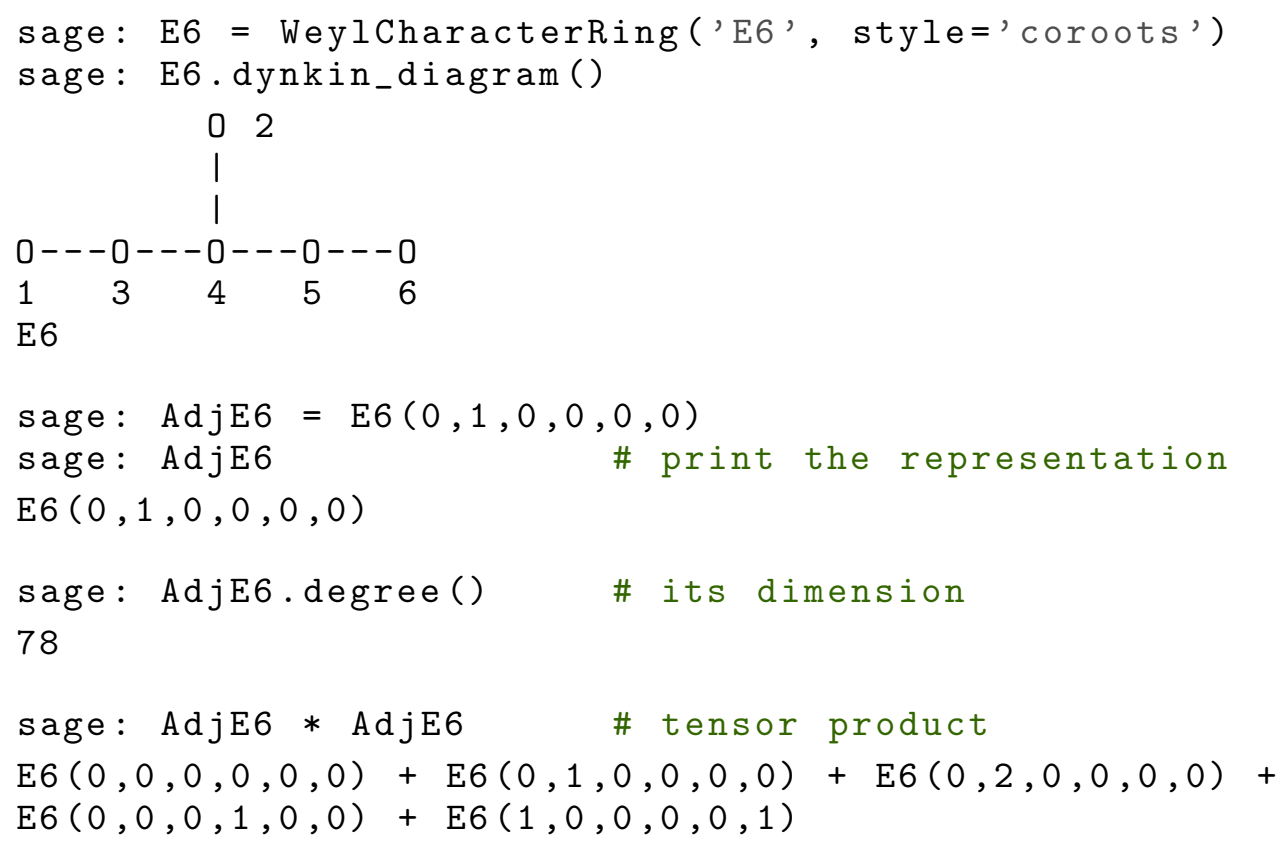

Here, we used the coroots (a.k.a. Dynkin labels), which are non-negative integers corresponding to the nodes of the Dynkin diagram, to define the representation AdjE6. The branch() method computes the branching of the representation to a subgroup. In simple cases it will be able to guess the desired branching rule, for example the Levi-type branching rule associated to removing a node from the Dynkin diagram:

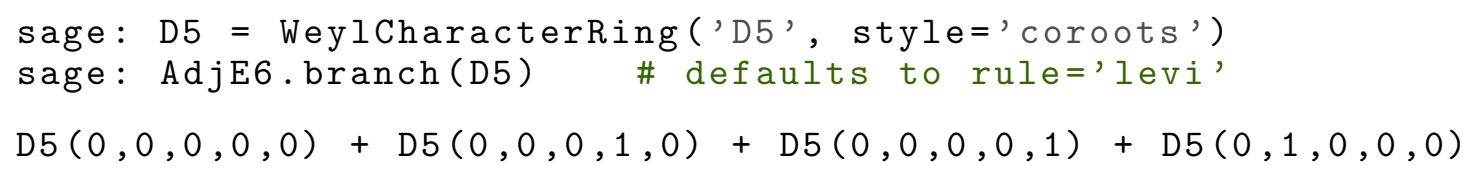

However, in general you have to specify it with the rule=<rulename $>$ keyword option. For example, applying the Levi-type rule twice: ${ }^{24}$

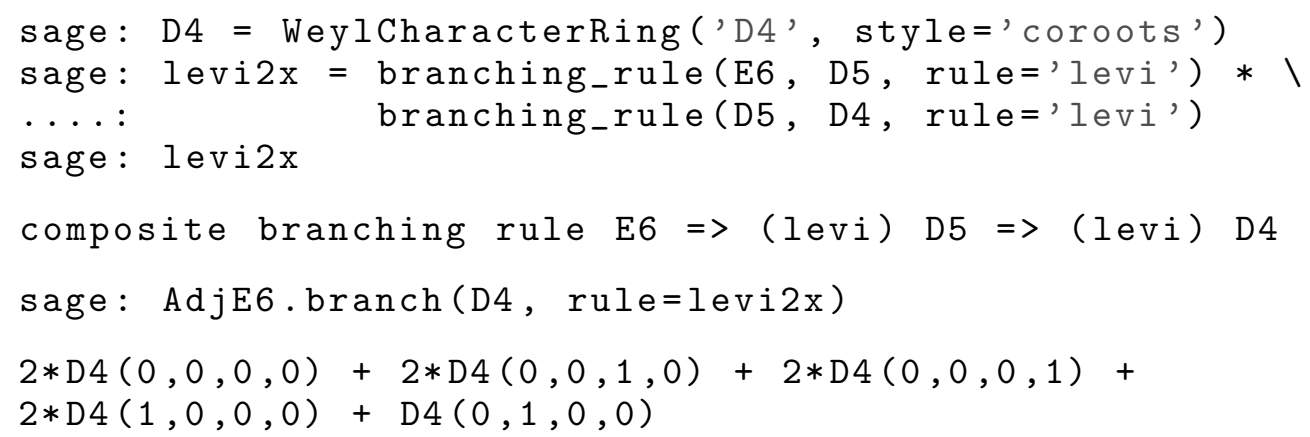

${ }^{24}$ Requires Sage version 6.1 or later. 


\begin{tabular}{|c|ccccccccc|}
\hline$G$ & $A_{n}$ & $B_{n}$ & $C_{n}$ & $D_{n}$ & $G_{2}$ & $F_{4}$ & $E_{6}$ & $E_{7}$ & $E_{8}$ \\
\hline$\lambda_{G}$ & 1 & 2 & 1 & 2 & 2 & 6 & 6 & 12 & 60 \\
\hline
\end{tabular}

Table 6. Extra normalization factor in the fundamental trace.

In addition to the Levi-type branching rules, there is a variety of other ones implemented. In particular, all branchings to maximal subgroups are. As a fancy example, here is the (non-maximal) branching rule associated to the automorphism of the $D_{4}$ Dynkin diagram:

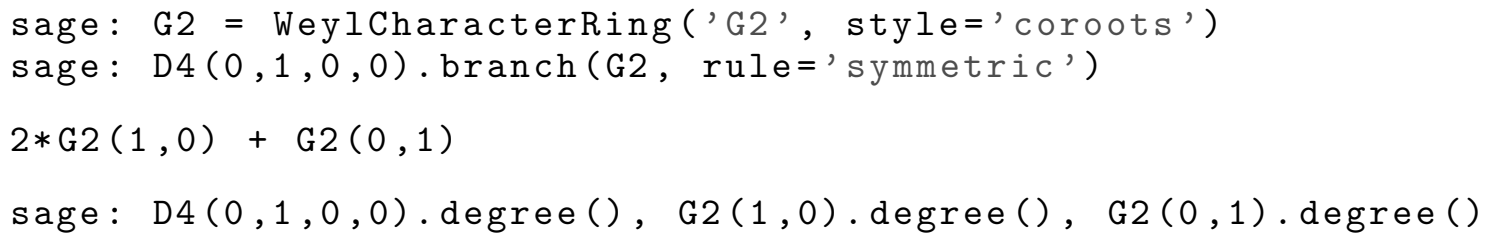

So the irreducible representations decompose as $\operatorname{ad}\left(D_{4}\right)=2 \times \underline{\mathbf{7}} \oplus \underline{\mathbf{1 4}}$ for this particular embedding $D_{4} \supset G_{2}$. Each representation can be restricted to a maximal torus $T \subset$ $G$, where it decomposes further into a sum of one-dimensional representations since $T$ is Abelian. These are the weights of the representation, and can be enumerated using

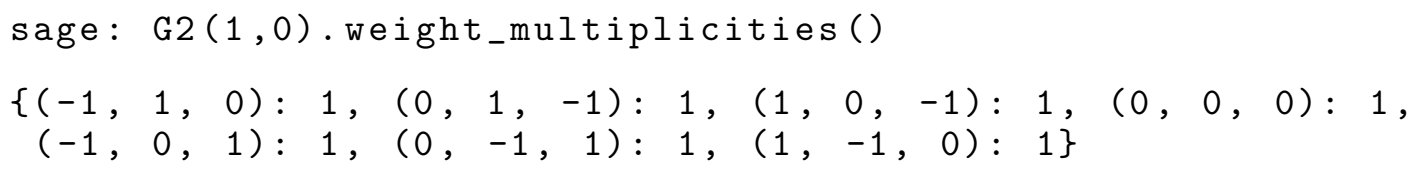

In particular, we can read off the multiplicity of the trivial weight having multiplicity 1 in $\underline{7}$. The number of all non-trivial weights is the charged dimension $[59,60]$, and we just computed that the charged dimension of $\underline{\mathbf{7}}$ is 6 .

Finally, one would like to know the anomaly coefficients. These are integers $A_{R}, B_{R}$, and $C_{R}$ associated to representations $R$ such that ${ }^{25}$

$$
\operatorname{tr}_{R} F^{2}=A_{R} \frac{1}{\lambda_{G}} \operatorname{tr} F^{2}, \quad \operatorname{tr}_{R} F^{4}=B_{R} \frac{1}{\lambda_{G}} \operatorname{tr} F^{4}+C_{R}\left(\frac{1}{\lambda_{G}} \operatorname{tr} F^{2}\right)^{2},
$$

where $\operatorname{tr}_{R}$ is the trace over the Lie algebra generator in the representation $R$ and $\operatorname{tr}$ is the trace over the fundamental representation. We will be using the integral normalization [60] where the factors $\lambda_{G}$ listed in table 6 are absorbed into the anomaly coefficients. That is, we use the properly normalized $\frac{1}{\lambda_{G}}$ tr for the trace over the fundamental representation in eq. (A.1). However, note that much of the physics literature uses the convention where the $\lambda_{G}$ prefactors are not absorbed. This then requires compensating factors of $\lambda_{G}$ in the gauge anomaly cancellation condition, which is why we are not following this convention. The traces on the right hand sides of eq. (A.1) are easily determined by restriction, that is, branching to a subgroup. We will be using $\mathrm{SU}(4)$, the simplest group with a quartic Casimir. There are various bases one can use for the representations of SU(4), for example

\footnotetext{
${ }^{25}$ If the group $G$ does not have an independent quartic Casimir operator then the two parameters $B_{R}, C_{R}$ are not uniquely determined. It is customary to define $B_{R}=0$ in that case. The computation of the anomaly coefficients is analogous but simpler, and it suffices to just consider a $\mathrm{SU}(2) \subset G$ subgroup instead of $\mathrm{SU}(4)$.
} 
- Irreducible representation $R(i, j, k)$ indexed by coroots,

- Tensor products $\underline{4}^{i} \otimes \underline{\mathbf{6}}^{j} \otimes \underline{\overline{4}}^{k}$, and

- Tensor products of symmetrizations

$$
S(i, j, k) \stackrel{\text { def }}{=} \operatorname{Sym}^{i}(\underline{\mathbf{4}}) \otimes \operatorname{Sym}^{j}(\underline{\mathbf{6}}) \otimes \operatorname{Sym}^{k}(\underline{\overline{4}}) .
$$

In either basis one can explicitly construct representations in terms of tensor operations. The traces for irreducible representations of the subgroup can then be evaluated directly. The third basis has the advantage that, for the two particular Lie algebra generators

$$
F_{1}=\frac{1}{\sqrt{2}} \operatorname{diag}(+1,-1,0,0), \quad F_{2}=\frac{1}{\sqrt{2}} \operatorname{diag}(0,0,+1,-1)
$$

of $\mathrm{SU}(4)$, one can explicitly evaluate the traces as polynomials in $i, j$, and $k$. They are

$$
\begin{aligned}
\operatorname{tr}_{S(i, j, k)} & F^{2}=\frac{1}{1814400} \prod_{x=1}^{3}(i+x) \prod_{y=1}^{5}(j+y) \prod_{z=1}^{3}(k+z) \\
\times & \left(21 i^{2}+20 j^{2}+21 k^{2}+84 i+120 j+84 k\right) \\
\operatorname{tr}_{S(i, j, k)} & F^{4}=\frac{1}{1814400} \prod_{x=1}^{3}(i+x) \prod_{y=1}^{5}(j+y) \prod_{z=1}^{3}(k+z) \\
\times & \left(30 i^{4}+60 i^{2} j^{2}+25 j^{4}+63 i^{2} k^{2}+60 j^{2} k^{2}+30 k^{4}+240 i^{3}+360 i^{2} j+240 i j^{2}\right. \\
& +300 j^{3}+252 i^{2} k+240 j^{2} k+252 i k^{2}+360 j k^{2}+240 k^{3}+435 i^{2} \\
& \left.+1440 i j+825 j^{2}+1008 i k+1440 j k+435 k^{2}-180 i-450 j-180 k\right) \\
\operatorname{tr}_{S(i, j, k)} & F_{1}^{2} F_{2}^{2}=\frac{1}{163296000} \prod_{x=1}^{3}(i+x) \prod_{y=1}^{5}(j+y) \prod_{z=1}^{3}(k+z) \\
\times & \left(45 i^{4}+180 i^{2} j^{2}+125 j^{4}+189 i^{2} k^{2}+180 j^{2} k^{2}+45 k^{4}+360 i^{3}+1080 i^{2} j\right. \\
& +720 i j^{2}+1500 j^{3}+756 i^{2} k+720 j^{2} k+756 i k^{2}+1080 j k^{2}+360 k^{3} \\
& \left.+495 i^{2}+4320 i j+4525 j^{2}+3024 i k+4320 j k+495 k^{2}-900 i+150 j-900 k\right) .
\end{aligned}
$$

Since the (usually reducible) representations $S(i, j, k)$ form a basis, one can express any representation as a linear combination of them. The anomaly coefficients then are

$$
\begin{aligned}
A_{R} & =\lambda_{G} \frac{\operatorname{tr}_{R} F_{1}^{2}}{\operatorname{tr} F_{1}^{2}}=\lambda_{G} \frac{\operatorname{tr}_{R} F_{2}^{2}}{\operatorname{tr} F_{2}^{2}} \\
B_{R} & =\lambda_{G} \frac{\operatorname{tr}_{R} F_{1}^{4}-3 \operatorname{tr}_{R} F_{1}^{2} F_{2}^{2}}{\operatorname{tr} F_{1}^{4}}=\lambda_{G} \frac{\operatorname{tr}_{R} F_{2}^{4}-3 \operatorname{tr}_{R} F_{1}^{2} F_{2}^{2}}{\operatorname{tr} F_{2}^{4}} \\
C_{R} & =\lambda_{G}^{2} \frac{3 \operatorname{tr}_{R} F_{1}^{2} F_{2}^{2}}{\left(\operatorname{tr} F_{1}^{2}\right)\left(\operatorname{tr} F_{2}^{2}\right)}
\end{aligned}
$$

We implemented the above algorithm in Sage, which one can find and use at the following URL: 


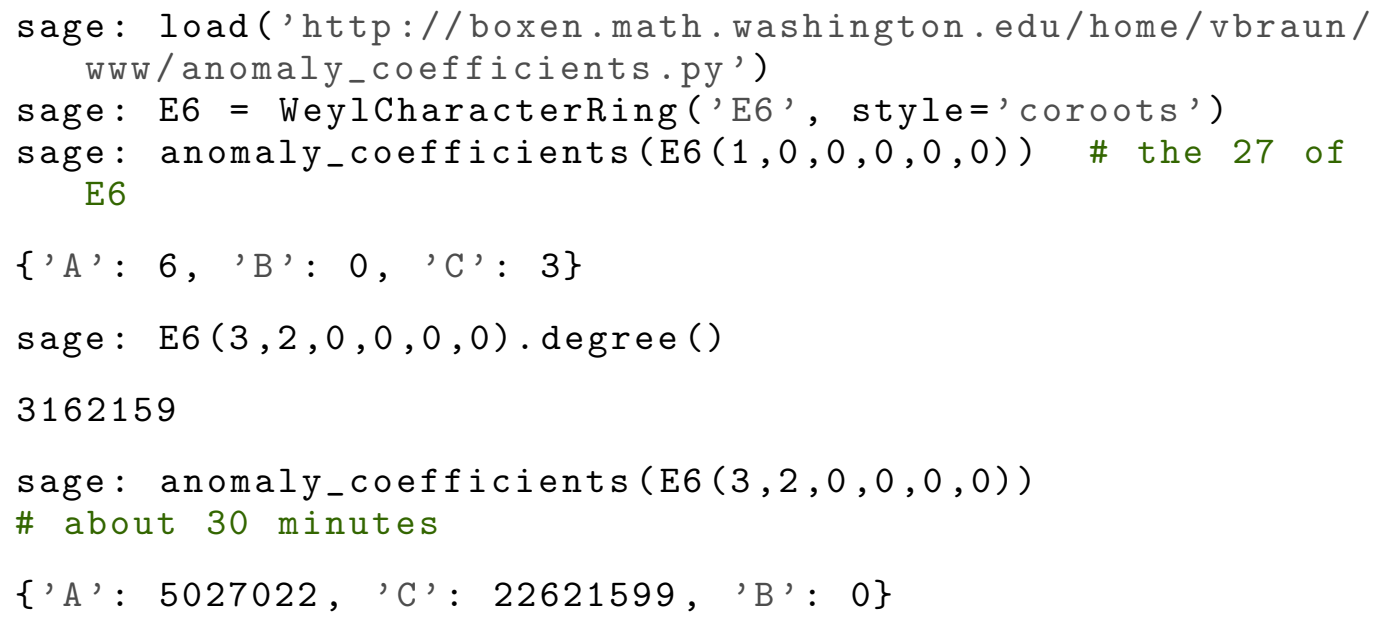

The group $D_{4}=\operatorname{Spin}(8)$ is special in that it has three independent Casimir operators in degree 4 . There are two ways to handle this, either by introducing an additional anomaly coefficient and another gauge anomaly cancellation condition or by demanding that the $F^{4}$-anomaly still cancels if one acts by triality. Note that the $B_{R}, C_{R}$ anomaly coefficients transform non-trivially under triality:

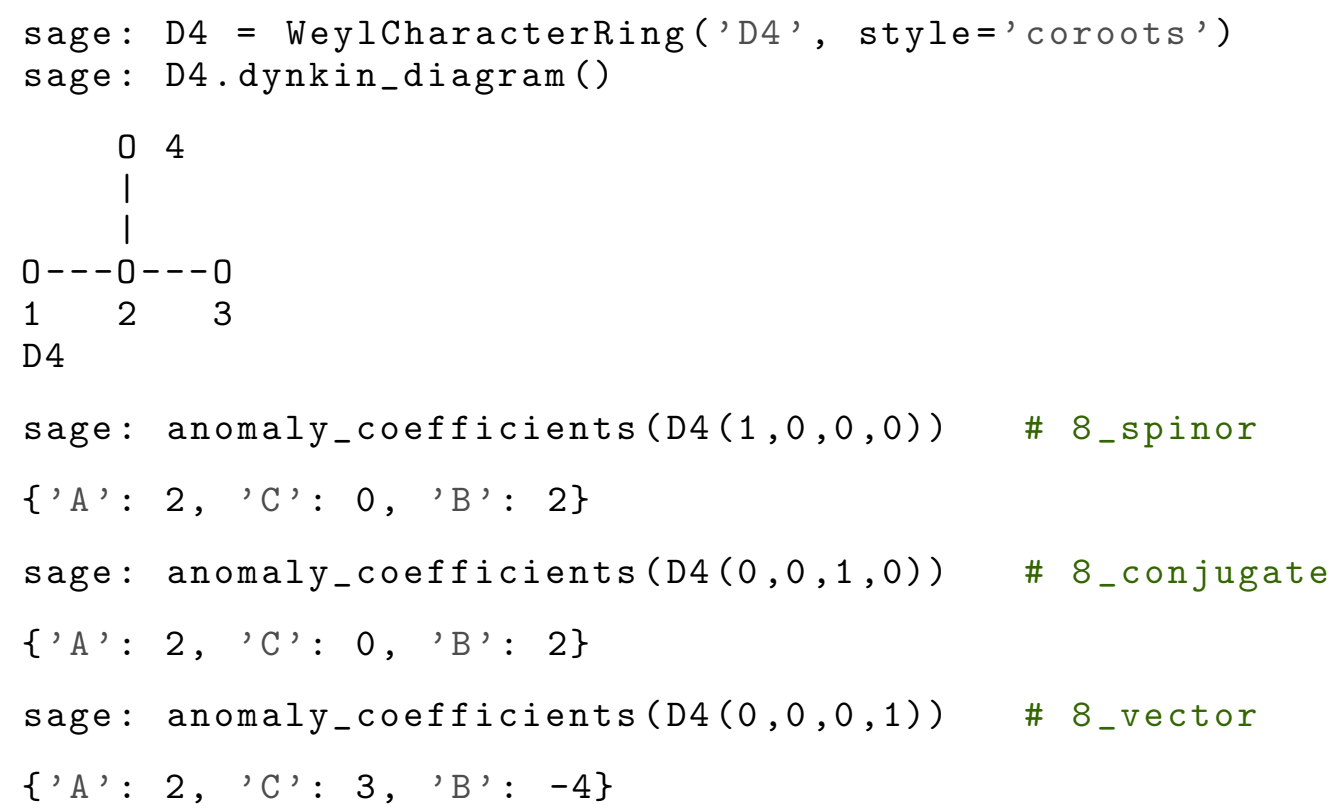

As a final example, consider the $\mathrm{SU}(N)$-representation $\square$, that is, with coroots $(0,2,0, \ldots, 0)$. Knowing that the anomaly coefficients are polynomials in $N$, we can easily determine them (see also table 1 of [74]):

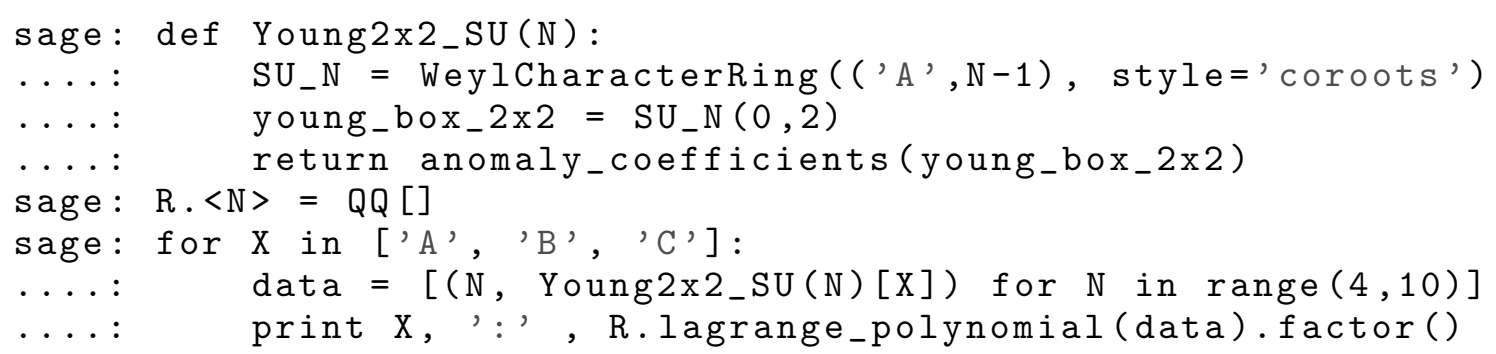




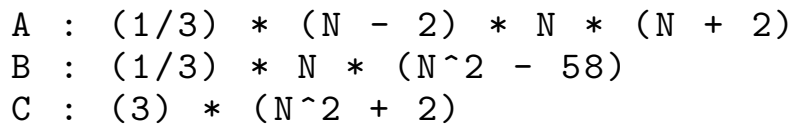

Open Access. This article is distributed under the terms of the Creative Commons Attribution License (CC-BY 4.0), which permits any use, distribution and reproduction in any medium, provided the original author(s) and source are credited.

\section{References}

[1] C. Vafa, Evidence for F-theory, Nucl. Phys. B 469 (1996) 403 [hep-th/9602022] [INSPIRE].

[2] D.R. Morrison and C. Vafa, Compactifications of F-theory on Calabi-Yau threefolds. 1, Nucl. Phys. B 473 (1996) 74 [hep-th/9602114] [InSPIRE].

[3] D.R. Morrison and C. Vafa, Compactifications of F-theory on Calabi-Yau threefolds. 2., Nucl. Phys. B 476 (1996) 437 [hep-th/9603161] [INSPIRE].

[4] J.H. Schwarz, An $\mathrm{SL}(2, \mathbb{Z})$ multiplet of type IIB superstrings, Phys. Lett. B 360 (1995) 13 [Erratum ibid. B 364 (1995) 252] [hep-th/9508143] [INSPIRE].

[5] P.S. Aspinwall, Some relationships between dualities in string theory, Nucl. Phys. Proc. Suppl. 46 (1996) 30 [hep-th/9508154] [INSPIRE].

[6] K. Kodaira, On compact analytic surfaces. II, Ann. Math. 77 (1963) 563.

[7] K. Kodaira, On compact analytic surfaces. III, Ann. Math. 78 (1963) 1.

[8] N. Nakayama, On Weierstrass models, in Algebraic geometry and commutative algebra, vol. II, Kinokuniya, Japan (1988), pg. 405.

[9] I. Dolgachev and M. Gross, Elliptic threefolds. I. Ogg-Shafarevich theory, J. Algebraic Geom. 3 (1994) 39 [alg-geom/9210009].

[10] E. Witten, Toroidal compactification without vector structure, JHEP 02 (1998) 006 [hep-th/9712028] [INSPIRE].

[11] J. de Boer et al., Triples, fluxes and strings, Adv. Theor. Math. Phys. 4 (2002) 995 [hep-th/0103170] [INSPIRE].

[12] V. Bouchard and H. Skarke, Affine Kac-Moody algebras, CHL strings and the classification of tops, Adv. Theor. Math. Phys. 7 (2003) 205 [hep-th/0303218] [INSPIRE].

[13] P. Berglund, J.R. Ellis, A.E. Faraggi, D.V. Nanopoulos and Z. Qiu, Elevating the free fermion $\mathbb{Z}_{2} \times \mathbb{Z}_{2}$ orbifold model to a compactification of F-theory, Int. J. Mod. Phys. A 15 (2000) 1345 [hep-th/9812141] [INSPIRE].

[14] S. Lang and J. Tate, Principal homogeneous spaces over abelian varieties, Amer. J. Math. 80 (1958) 659.

[15] I.R. Šafarevič, Principal homogeneous spaces defined over a function field, Trudy Mat. Inst. Steklov. 64 (1961) 316 [AMS Transl. 37 (1964) 85].

[16] N. Nakayama, Global structure of an elliptic fibration, Publ. Res. Inst. Math. Sci. 38 (2002) 451.

[17] R. Donagi and T. Pantev, Torus fibrations, gerbes and duality, Mem. Amer. Math. Soc. 193 (2008) vi+90 [math. AG/0306213] [INSPIRE]. 
[18] D.R. Morrison, Wilson lines in F-theory, lecture at Harvard University, unpublished, U.S.A. January 81999.

[19] P. Deligne, Courbes elliptiques: formulaire (d'après J. Tate), in Modular functions of one variable, IV (Proc. Internat. Summer School, Univ. Antwerp, Antwerp Belgium 1972), Lect. Notes Math. 476, Springer, Berlin Germany (1975), pg. 53.

[20] D. Mumford and K. Suominen, Introduction to the theory of moduli, in Algebraic geometry, Oslo Norway 1970 (Proc. Fifth Nordic Summer-School in Math.), Wolters-Noordhoff, The Netherlands (1972), pg. 171.

[21] S.Y. An et al., Jacobians of genus one curves, J. Number Theory 90 (2001) 304.

[22] M. Artin, F. Rodriguez-Villegas and J. Tate, On the Jacobians of plane cubics, Adv. Math. 198 (2005) 366.

[23] A. Weil, Remarques sur un mémoire d'Hermite (in French), Arch. Math. (Basel) 5 (1954) 197.

[24] J.J. Duistermaat, Discrete integrable systems. QRT maps and elliptic surfaces, Springer Monographs in Mathematics, Springer, Berlin Germany (2010), pg. xxii+627.

[25] D.R. Morrison and D.S. Park, F-theory and the Mordell-Weil group of elliptically-fibered Calabi-Yau threefolds, JHEP 10 (2012) 128 [arXiv:1208.2695] [INSPIRE].

[26] G. Salmon, A treatise on the analytic geometry of three dimensions, vol. $1,7^{\text {th }}$ ed., C.H. Rowe ed., Chelsea Publishing Company, New York U.S.A. (1958).

[27] Sage Development Team collaboration, V. Braun and J. Keitel, Weierstrass form for complete intersection of two quadratic equations, http://trac.sagemath.org/14855, (2013).

[28] D.A. Buchsbaum and D. Eisenbud, Algebra structures for finite free resolutions, and some structure theorems for ideals of codimension 3, Amer. J. Math. 99 (1977) 447.

[29] T. Fisher, The invariants of a genus one curve, Proc. Lond. Math. Soc. 97 (2008) 753 [math.NT/0610318].

[30] I. Dolgachev and A. Libgober, On the fundamental group of the complement to a discriminant variety, in Algebraic geometry (Chicago U.S.A. 1980), Lect. Notes Math. 862, Springer, Berlin Germany (1981), pg. 1.

[31] R. Miranda, Smooth models for elliptic threefolds, in The birational geometry of degenerations (Cambridge U.S.A. 1981), Progr. Math. 29, Birkhäuser, Boston U.S.A. (1983), pg. 85.

[32] M. Buican, D. Malyshev, D.R. Morrison, H. Verlinde and M. Wijnholt, D-branes at singularities, compactification and hypercharge, JHEP 01 (2007) 107 [hep-th/0610007] [INSPIRE].

[33] Y. Kawamata, K. Matsuda and K. Matsuki, Introduction to the minimal model problem, in Algebraic geometry, Sendai Japan 1985, Adv. Stud. Pure Math. 10, North-Holland, The Netherlands (1987), pg. 283.

[34] A. Grothendieck, Le groupe de Brauer, I, II, III (in French), in Dix Exposés sur la Cohomologie des Schémas, North-Holland, The Netherlands (1968), pg. 46.

[35] P.S. Aspinwall, D.R. Morrison and M. Gross, Stable singularities in string theory, Commun. Math. Phys. 178 (1996) 115 [hep-th/9503208] [inSPIRE].

[36] C. Vafa and E. Witten, On orbifolds with discrete torsion, J. Geom. Phys. 15 (1995) 189 [hep-th/9409188] [INSPIRE]. 
[37] F. Denef, Lectures on constructing string vacua, in String theory and the real world: from particle physics to astrophysics, C. Bachas ed., Elsevier, The Netherlands (2008), pg. 483 [arXiv:0803.1194] [INSPIRE].

[38] B.R. Greene, A.D. Shapere, C. Vafa and S.-T. Yau, Stringy cosmic strings and noncompact Calabi-Yau manifolds, Nucl. Phys. B 337 (1990) 1 [InSPIRE].

[39] M. Gross and P.M.H. Wilson, Large complex structure limits of K3 surfaces, J. Diff. Geom. 55 (2000) 475 [math.DG/0008018].

[40] J. Marsano, N. Saulina and S. Schäfer-Nameki, On G-flux, M5 instantons and U(1)s in F-theory, arXiv:1107.1718 [INSPIRE].

[41] V.V. Batyrev, Dual polyhedra and mirror symmetry for Calabi-Yau hypersurfaces in toric varieties, J. Alg. Geom. 3 (1994) 493 [alg-geom/9310003] [INSPIRE].

[42] V. Braun, Toric elliptic fibrations and F-theory compactifications, JHEP 01 (2013) 016 [arXiv:1110.4883] [INSPIRE].

[43] M. Kreuzer and H. Skarke, On the classification of reflexive polyhedra, Commun. Math. Phys. 185 (1997) 495 [hep-th/9512204] [INSPIRE].

[44] A. Grassi and V. Perduca, Weierstrass models of elliptic toric K3 hypersurfaces and symplectic cuts, Adv. Theor. Math. Phys. 17 (2013) 741 [arXiv:1201.0930] [INSPIRE].

[45] P. Candelas and H. Skarke, F theory, SO(32) and toric geometry, Phys. Lett. B 413 (1997) 63 [hep-th/9706226] [inSPIRE].

[46] P. Candelas, E. Perevalov and G. Rajesh, Matter from toric geometry, Nucl. Phys. B 519 (1998) 225 [hep-th/9707049] [INSPIRE].

[47] P. Candelas, E. Perevalov and G. Rajesh, Toric geometry and enhanced gauge symmetry of F-theory/heterotic vacua, Nucl. Phys. B 507 (1997) 445 [hep-th/9704097] [InSPIRE].

[48] P. Candelas, E. Perevalov and G. Rajesh, Comments on A, B, C chains of heterotic and type-II vacua, Nucl. Phys. B 502 (1997) 594 [hep-th/9703148] [INSPIRE].

[49] P. Candelas, A. Constantin and H. Skarke, An abundance of $K 3$ fibrations from polyhedra with interchangeable parts, Commun. Math. Phys. 324 (2013) 937 [arXiv:1207.4792] [INSPIRE].

[50] V. Braun, T.W. Grimm and J. Keitel, New global F-theory GUTs with U(1) symmetries, JHEP 09 (2013) 154 [arXiv: 1302.1854] [INSPIRE].

[51] V. Braun, T.W. Grimm and J. Keitel, Geometric engineering in toric F-theory and GUTs with U(1) gauge factors, JHEP 12 (2013) 069 [arXiv:1306.0577] [INSPIRE].

[52] Y. Hu, C.-H. Liu and S.-T. Yau, Toric morphisms and fibrations of toric Calabi-Yau hypersurfaces, Adv. Theor. Math. Phys. 6 (2003) 457 [math/0010082] [InSPIRE].

[53] Sage Development Team collaboration, V. Braun and A.Y. Novoseltsev, Toric varieties framework for Sage, http://sagemath.org/doc/reference/sage/schemes/toric/variety.html, (2010).

[54] M. Cvetič, D. Klevers and H. Piragua, F-theory compactifications with multiple U(1)-factors: constructing elliptic fibrations with rational sections, JHEP 06 (2013) 067 [arXiv:1303.6970] [INSPIRE].

[55] M. Cvetič, A. Grassi, D. Klevers and H. Piragua, Chiral four-dimensional F-theory compactifications with $\mathrm{SU}(5)$ and multiple $\mathrm{U}(1)$-factors, JHEP 04 (2014) 010 [arXiv: 1306.3987] [INSPIRE]. 
[56] M. Cvetič, D. Klevers and H. Piragua, F-theory compactifications with multiple U(1)-factors: addendum, JHEP 12 (2013) 056 [arXiv: 1307.6425] [INSPIRE].

[57] M. Cvetič, D. Klevers, H. Piragua and P. Song, Elliptic fibrations with rank three Mordell-Weil group: F-theory with $\mathrm{U}(1) \times \mathrm{U}(1) \times \mathrm{U}(1)$ gauge symmetry, JHEP 03 (2014) 021 [arXiv: 1310.0463] [INSPIRE].

[58] D.S. Park and W. Taylor, Constraints on $6 D$ supergravity theories with Abelian gauge symmetry, JHEP 01 (2012) 141 [arXiv:1110.5916] [INSPIRE].

[59] A. Grassi and D.R. Morrison, Group representations and the Euler characteristic of elliptically fibered Calabi-Yau threefolds, J. Alg. Geom. 12 (2003) 321 [IASSNS-HEP-00/27] [math. AG/0005196 [INSPIRE].

[60] A. Grassi and D.R. Morrison, Anomalies and the Euler characteristic of elliptic Calabi-Yau threefolds, Commun. Num. Theor. Phys. 6 (2012) 51 [arXiv:1109.0042] [InSPIRE].

[61] E. Witten, Phase transitions in M-theory and F-theory, Nucl. Phys. B 471 (1996) 195 [hep-th/9603150] [INSPIRE].

[62] H. Hayashi, C. Lawrie, D.R. Morrison and S. Schäfer-Nameki, Box graphs and singularities, to appear.

[63] M. Bershadsky et al., Geometric singularities and enhanced gauge symmetries, Nucl. Phys. B 481 (1996) 215 [hep-th/9605200] [INSPIRE].

[64] J. Tate, Algorithm for determining the type of a singular fiber in an elliptic pencil, in Modular functions of one variable, IV (Proc. Internat. Summer School, Univ. Antwerp, Antwerp Belgium 1972), Lect. Notes Math. 476, Springer, Berlin Germany (1975), pg. 33.

[65] S. Katz, D.R. Morrison, S. Schäfer-Nameki and J. Sully, Tate's algorithm and F-theory, JHEP 08 (2011) 094 [arXiv: 1106.3854] [InSPIRE].

[66] E. Witten, New 'gauge' theories in six-dimensions, JHEP 01 (1998) 001 [hep-th/9710065] [INSPIRE].

[67] A. Degeratu and K. Wendland, Friendly giant meets pointlike instantons? On a new conjecture by John McKay, in Moonshine - The First Quarter Century and Beyond, A Workshop on the Moonshine Conjectures and Vertex Algebras, LMS Lect. Notes Ser. $\mathbf{3 7 2}$, London Mathematical Society, London U.K. (2010).

[68] T. Shioda, On elliptic modular surfaces, J. Math. Soc. Japan 24 (1972) 20.

[69] J. Tate, Algebraic cycles and poles of zeta functions, in Arithmetical Algebraic Geometry (Proc. Conf. Purdue Univ., U.S.A. 1963), Harper \& Row, U.S.A. (1965), pg. 93.

[70] J. Tate, On the conjectures of Birch and Swinnerton-Dyer and a geometric analog, in Séminaire Bourbaki, vol. 9, Soc. Math. France, France (1995), pg. 415.

[71] R. Wazir, Arithmetic on elliptic threefolds, Composit. Math. 140 (2004) 567 [math.NT/0112259].

[72] Sage Development Team collaboration, W.A. Stein et al., Sage Mathematics Software (Version 6.1), http://www.sagemath.org/, (2012).

[73] Sage-Combinat community collaboration, Sage-Combinat: enhancing Sage as a toolbox for computer exploration in algebraic combinatorics, http://combinat.sagemath.org/, (2012).

[74] V. Kumar, D.S. Park and W. Taylor, 6D supergravity without tensor multiplets, JHEP 04 (2011) 080 [arXiv:1011.0726] [INSPIRE]. 